\title{
Knowledge management in SMEs and MNCs: Matching knowledge mobility mechanisms to supply network configuration profiles
}

\author{
Tomás Seosamh Harrington a , Jagjit Singh Srai ${ }^{b}$ and Mukesh Kumar ${ }^{b}$ \\ a Innovation, Technology and Operations Management Group, Norwich Business School, \\ Faculty of Social Sciences, University of East Anglia (UEA), UK \\ ${ }^{b}$ Centre for International Manufacturing, Institute for Manufacturing (IfM), University of \\ Cambridge, UK
}

\begin{abstract}
:
While 'knowledge mobility' presents significantly different challenges for SMEs and MNCs, it is strongly influenced by two common factors: the type of knowledge to be integrated and the configuration of the operations network. Only by understanding the various types of knowledge and how they - and the configuration profile of the network (Nascent, Emerging, or Mature; Local, Regional, or Global; SME or MNC) - affect how knowledge is shared, can a proactive and integrated approach to knowledge management be developed.

This study specifically examines knowledge transfer mechanisms in knowledgeintensive manufacturing firms and across their networks. Here, both SMEs and MNCs are required to make critical decisions about the 'level' of collaboration and knowledge sharing with network partners - whether it should be purely transactional or more strategic.

A network configuration framework is extended to incorporate knowledge-specific dimensions of analysis, derived from the academic literature on stages of emergence and knowledge transfer theory, and supported by a review of knowledge integration mechanisms in practice. This was supported by benchmarking exercises involving a series of multiorganisational network cases with different types of SME-SME and MNC-SME interactions, and gaining insights on factors that will affect future Knowledge Mobility Configurations through a series of industrial case studies. The resulting Knowledge Mobility Configuration (KMC) framework was tested and refined using five case studies indicative of a growing shift towards information and knowledge-intensive activities - involving production and supply network 'partners' - in both SME and MNC contexts.

In terms of contribution, there has been limited empirical research to-date into the determinants of successful knowledge transfers in MNC-SME network contexts. The network configuration element of the KMC framework provides insights on how such networks evolve, and how specific knowledge mobility profiles may evolve over time. Our findings demonstrate the critical role of knowledge management in internationalisation, and that skills, knowledge, technology and organisational processes are integral to any emerging network design criteria and/or 'capability' acquisition assessment. The choice of integration mechanism is not only
\end{abstract}


influenced by the type of knowledge being 'mobilised', but also by industrial context and the maturity of the network within which the knowledge is being shared. This more nuanced approach may be used as a basis for a proactive and differentiated approach to knowledge mobility and integration across SME and MNC networks.

Keywords: Knowledge Management; Knowledge Sharing; Knowledge Mobility; Network Configuration; MNCs; SMEs

\section{Introduction}

The importance of knowledge as a critical element of modern organisational performance has been widely reported in the academic literature (e.g. Grant, 1996; Novins and Armstrong, 1998; Drucker, 1999; Birkinshaw, 2001; Yang, 2008; Durst and Edvardsson, 2012; Aboelmaged, 2014). Collaborative networked organisations are emerging that seek access to new skills, knowledge, markets and technologies to meet emerging market needs - through sharing risk, integrating complementary competencies and leveraging specialist capabilities (Romero and Molina, 2011; Dooley, Kirk, and Philpott, 2013; Harrington and Srai, 2016)

This study looks to develop a knowledge management methodology that can be used by networked organisations and their partners, to exploit the synergies available from better network coordination. While advances in knowledge management usually focus on the needs of larger organizations (Gupta and Govindarajan 2000), there has been little systematic empirical investigation into the determinants of successful knowledge transfers in MNC-SME network contexts. Furthermore, while the benefits of implementing knowledge management strategies have been proven, research has largely focused on technology as a solution often neglecting critical relational factors (Harrington et al., 2012; Pawar and Rogers, 2014; Harrington et al., 2016).

For both SMEs and MNCs, an ability to identify and re-organise around future 'capability' requirements has also become increasingly important, especially where 'knowledge' plays a critical role in network partner selection and future organisational design (Harrington and Srai 2016). Inefficient use of resources, and ineffective operations and supply chain procedures are often cited as causes of failure for both SMEs and MNCs (Gold et al., 2001; Ates et al 2013; Cerchione et al, 2015, Gunasekaran et al, 2000; Lee and Klassen, 2008; Harrington and Srai 2017). While larger organisations, with a history of international operations, are much more familiar with the problems of knowledge integration, few - if any claim to have overcome those (Fleet et al., 2014).

A key determinant in better understanding the success or failure of any knowledge initiative is the identification and assessment of the context in which 'transfer' or 'sharing' takes place and the preconditions that are necessary for the effort to succeed (Gupta and Govindarajan, 2000; Gold et al, 2001). Organisations need to make decisions about the 'level' 
of collaboration and knowledge sharing that should exist between partners - whether it should be purely transactional or strategic (Harrington and Srai 2016).

An overview of knowledge transfer theory is presented to inform the development of a Knowledge Mobility Configuration (KMC) framework, which combines network configuration aspects of knowledge within multi-partnered contexts, with knowledge transfer mechanisms. Through analyzing and integrating different determinants of knowledge networks and how they support or enable sharing processes, this research contributes to the body of knowledge by providing a holistic understanding of the dimensions, their relationships, and the impact of different network configurations at various maturity levels. Network case studies, that were indicative of the growing shift towards knowledge-critical activities, were specifically selected to test and refine the KMC framework. The cases involved a series of regional suppliers (SMEs) and global manufacturing networks (MNCs), and enabled an examination of real knowledge sharing networks in practice today. The research also explored the relationship between management and engineers as well as comparing these opinions with the perceived ideals for knowledge mobility for production lines at various states of maturity. The KMC framework enables the 'matching' of optimal knowledge mobility mechanism(s) to specific supply network configuration profiles of MNCs and SMEs. It can provide practitioners with a visual representation of the current state of knowledge sharing within their organisation and wider networks, and an approach to assessing potential future states and mechanisms.

The paper is structured as follows. Section 2 introduces the research domains, providing background information, describing the research area and scope, and reviewing the existing literature on knowledge, knowledge mechanisms, and network configuration. Section 3 presents the prototype Knowledge Mobility Configuration framework, derived from outputs from the literature review. Section 4 summarises the research methodology and case studies. Section 5 reviews the findings from the study including application and test of the prototype framework for Knowledge Configuration Profiling. Section 6 summarises the final framework following refinement, and discusses instructive insights from the 'real' case studies. Section 7 concludes with a summary, identifying limitations and areas for further work.

\section{Literature review}

Good knowledge management practices integrates organisation, people, processes and technology with the ultimate goal of improving organisational performance (O'Dell and Grayson, 1998; Wiig 1999; Wang and Plaskoff, 2002). To better understand the critical dimensions of knowledge management in MNCs and SMEs, it is necessary to develop a clear picture of how various knowledge areas are related and how 'mobility' is facilitated or impeded by various configurational and other factors. This section summarises the key literature in the domains of knowledge mobility and network configuration to inform the development of the KMC framework. 


\subsection{Developing a Model of Knowledge Mobility}

Knowledge is integral to any emerging network design criteria (Harrington and Srai 2016). Activities related to knowledge management, including knowledge acquisition and knowledge sharing, are defined here as Knowledge Mobility (KMob). KMob is understood as the communication or shared understanding of knowledge (Szulanski, 2003), and is costly, time consuming, labour intensive and difficult, and requires a certain level of trust (Szulanski, 1996; Durst and Edvardsson, 2012; Landryová and Irgens, 2006; Liu et al, 2014; Hora \& Klassen, 2013; Argote et al, 1999; Harrington and Srai 2016). However, it is only by understanding the various types of knowledge - and how these affect how knowledge is shared - within the configuration profile of a network (Nascent, Emerging, or Mature; Local, Regional, or Global; SME or MNC) can a proactive and integrated approach to knowledge management be developed.

To aid frame the development of the KMC framework, Figure 1 illustrates a model for KMob showing key linkages in a manufacturing mobility context, and the key areas of focus for this research, namely:

- Network configuration and maturity

- MNC and SME perspectives on knowledge

- Knowledge types and knowledge mobility mechanisms

Manufacturing choices and knowledge influence the choice or specification of process equipment which may radically alter the specification of the production process, innovation capability, value network, and supply chain partners required in delivering an end product (Harrington et al., 2017; Delbufalo, 2017). In any assessment, decisions regarding the network also require a certain body of knowledge as changes in any decision area will affect choices 'upstream' and 'downstream', in addition to the application of appropriate knowledge management concepts locally and globally (Di Gregorio et al, 2009; Fleet et al., 2014). This is true for both MNCs and SME networks, irrespective of size and global reach. Hence, in the case of MNCs and SMEs, the type of knowledge being transferred largely influences the choice of knowledge mobility mechanism, as does the network configuration - and the maturity of the network - within which the knowledge is being mobilised or integrated. Sections 2.2-2.5 now examine KMob mechanisms in the context of network configuration and knowledge type. 


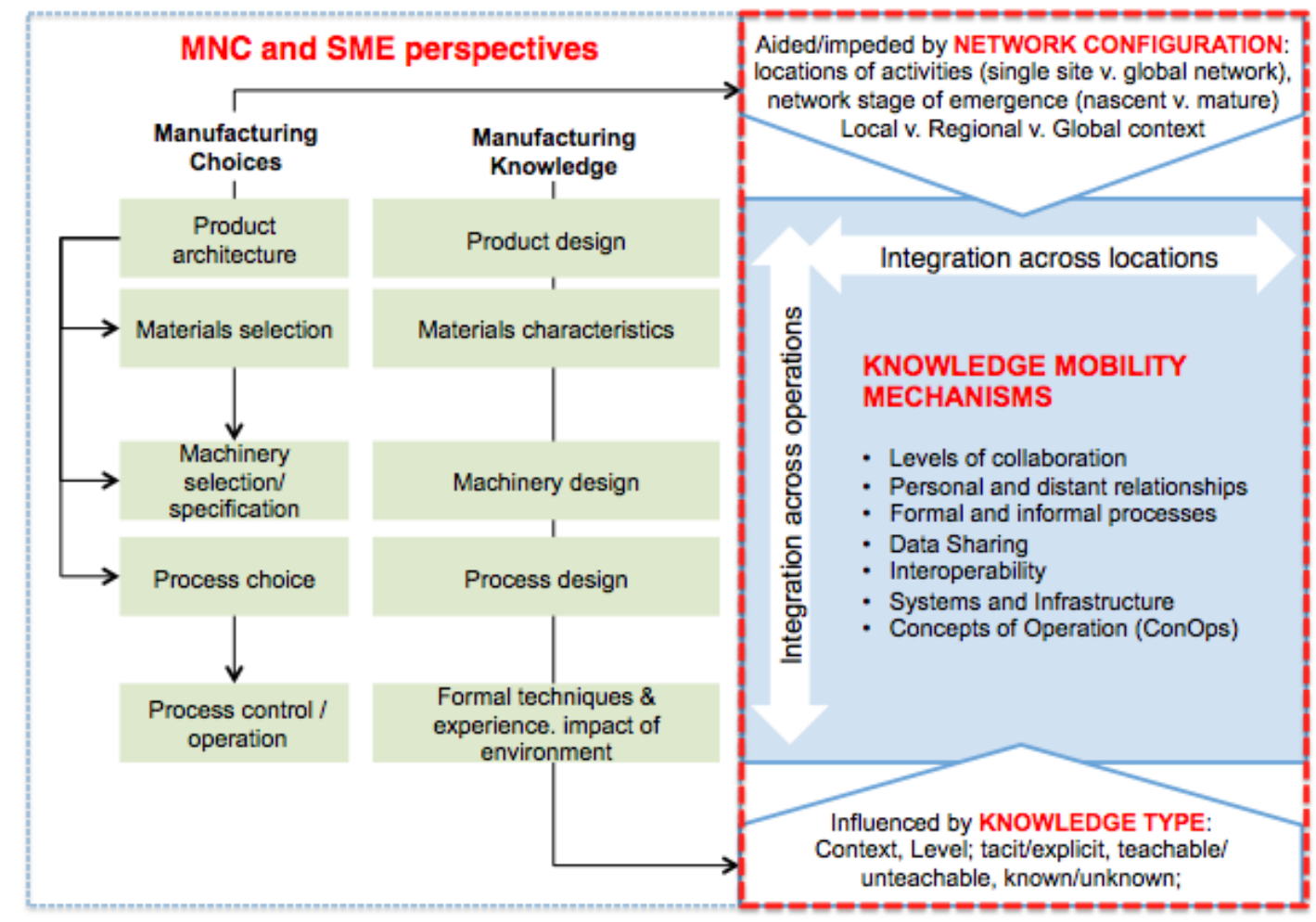

Figure 1. Model for Knowledge Mobility showing key linkages in a manufacturing mobility context (adapted from Grant 1999; Minshall 1999; Pongpanich 2000; Harrington et al., 2012; Fleet et al., 2014; Harrington and Srai 2016; Harrington and Srai 2017)

\subsection{Network Configuration}

Different types of network configurations - with distinguishable strategic objectives, specific target markets, critical resources and certain operational behaviours - differ based on their characteristics and purpose (Srai and Gregory, 2008). Van Waarden (1992) described networks, as patterns of relations between actors that are extremely dependent on the relationships that are in existence between the organisations involved and the structure of the networks in which they operate.

Product architecture is one such characteristic that has a strong influence on the configuration of the operations network, and by extension, the knowledge that needs to be managed and shared (Srai and Gregory, 2008). For SMEs and MNCs, architectures may range from weak, initial product focus and definition, to alpha-product development, to mature products. Nevertheless, organisations have some degrees of freedom (options) in selecting and reconfiguring their network configuration, and they may have more or less well-developed capabilities and mature networks to manage it (Harrington et al., 2012). This is an area of growing importance, given the argument that a supply network never reaches true 'maturity' (Harrington and Srai, 2017). One reason for this is the increasing dynamism with which today's 'industrial enterprise' is engaging with recent advances in technology, for example, 
the Internet of Things (IOT) and digitalisation (Harrington and Srai 2016). Here, organisations need to make decisions about the 'level' of collaboration and knowledge sharing that should exist between partners. Hence, 'capability' features such as skills, knowledge, technology and organisational processes are integral to any emerging network design criteria and/or capability acquisition assessment (Harrington et al., 2015; Harrington and Srai 2016).

\subsubsection{Network Maturity}

Innovation and knowledge transfer has moved from a corporate model of knowledge production towards a new distributed, inter-organisational, innovation model (Thether, 2005; Hewitt-Dundas, 2012), where certain environments will support a learning culture more than others. Characteristics such as networks with an entrepreneurial, learning or innovation focus, having the stability or tendency to change, and facilitate the mobility of personnel can show very different impacts (Cummings 2003). By examining knowledge mobility mechanisms within the context of network maturity, this research examines how both the knowledge task and also the available knowledge mobility mechanism are modified by network maturity and industry context. This more nuanced approach may be used as a basis for a proactive and differentiated approach to knowledge integration within the network.

Knowledge management, mediated by innovation, can have an effect on operations performance (Aboelmaged, 2014). Different approaches to the classification of phases and stages have been widely reported in the academic literature from an innovation perspective. (Utterback and Abernathy 1975; Rogers 2003; Hansen and Birkinshaw 2007). Although there are some approaches which pertain to a network view (in terms of business structure and organisational archetype) few have considered the role of network configuration and maturity, in the context of managing knowledge. As here are significant structural differences between SMEs and MNCs, this study on knowledge mobility considers networks in three distinct 'phases' of emergence linked to network configuration dimensions and sub-dimensions, derived for nascent and emerging contexts from the academic literature (Harrington and Srai 2017). From a knowledge perspective, different knowledge types at each stage will require tailored mechanisms for transfer for both MNCs and SMEs- see figure 2. 


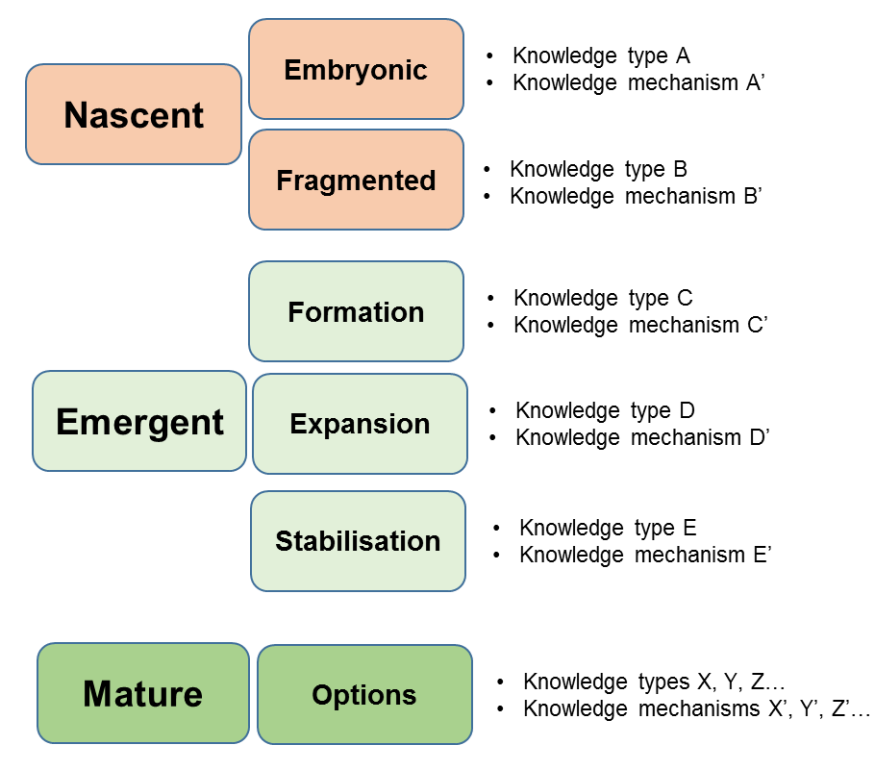

Figure 2: Phases and sub-phases of supply network emergence (adapted from Harrington and Srai, 2012, 2017) - different knowledge types at each stage will require tailored mechanisms for transfer

\subsection{SME and MNC perspectives}

Even though the results are often difficult to quantify (Davenport, 2002), the benefits of improved knowledge management within organisations, and knowledge integration across partner networks, is well documented in the literature (Deitz and Ellershaw 1999; Durst and Edvardsson, 2012; Harrington et al., 2012). In practice, only a few organisations who have identified the benefits of $\mathrm{KM}$ and are actively working on establishing in-house $\mathrm{KM}$ solutions believe that they were accomplished at processes within their organisation (Ruggles, 1998).

The adoption of new knowledge from external sources, and from other industries, is a growing source of innovation (Alexander and Childe, 2012; Phillips, Harrington and Srai, 2017). Hence, the ability to effective mobilise and integrate knowledge across global value networks of diverse specialist players is arguably becoming a strategic differentiator (Fleet et al., 2014; Liu et al, 2014). Here, it is crucial for both MNCs and SMEs to invest in KM, as great competitive advantage can be achieved by managing knowledge between their units and subsidiaries, and efficiently combine knowledge from global resource pools (Doz and Prahalad 1991). It is challenging to establish such an environment for effective information and knowledge exchange as there is no "one size fits all" policy and what might work in one company or one subsidiary might not necessarily be as effective in another (Lucas, 2006). 
For single-site SMEs many issues of knowledge integration may be dealt with relatively simply, and even informally (Fleet, et al., 2014). Information in the production function may be passed between different teams or shifts by face-to-face contact (shift change-over meetings, for example) or through log books and documentation. Integration between different functions is similarly straightforward and may entail the exchange of documents and emails. SMEs tend to consider their flexibility and ability to react quickly to customer needs as a competitive advantage in comparison to large firms (Carson \& Gilmore, 2000). In the context of organisations and the wider network, Ambrose et al (2010) have investigated predictors of success in high value buyer supplier relationships and suggest that communication is the sole significant factor. For SMEs engaging for the multi-organisational network partnering for the first time, proximity helps in communication and in quickly detecting and resolving misunderstanding. For SMEs internationalising for the first time, the problem of knowledge integration is often far more important than the concerns they have about specific issues regarding a new location. Linking knowledge and information in this context to concepts of communication and dependency, 'asymmetry' across SMEs and the participating parties often leads to uncertainty, resulting in a shorter-term orientation with SMEs who then focus less on key performance indicators, with longer-term effects on performance (Premkumar 2006; Ates et al., 2013). As social interactions and transfer of tacit knowledge is becoming more frequent than in the past, tailored transfer channels have become more important and there is a need to extend the performance indicators beyond commercial aspects like patenting, licensing and spin-offs. These latest mechanisms, although important, are an incomplete representation of the wide process of knowledge exchange (D'Este \& Patel, 2007), especially in the case of SMEs.

In a multinational company, knowledge integration may be viewed as being much more complicated. Sharing knowledge across different production sites is hampered not only by distance, potentially by language, culture and different equipment or operating conditions. Even Intel Corporation's "copy exactly!" philosophy - enabling delivery of product from multiple production sites (in effect operating as a "virtual factory" that performs consistently and independent of the manufacturing source site) - has exceptions to the rule - as some of the ways of doing things on one site may not work on another (author, personal communication). When a production process is first transferred from one site to another it may expose gaps in knowledge about critical conditions (such as humidity, temperature and air pressure) for the process that may previously have been taken for granted. If it is difficult to integrate knowledge across different sites performing the same function, it is arguably even more challenging to do so across multiple sites and different functions - given the divergent perspectives of the various functional groups (Fleet et al., 2014).

The twin concepts of "clustering" and "reach", often used when examining regional manufacturing capabilities or emerging industries (Schilling and Phelps, 2007) can be directly applied to assessing collaborative resources for MNC-SME networks. By measuring the number of links that each critical supplier has to others (clustering ability), and the distance 
through which information has to travel in order for exchange to occur (reach), it is possible to develop measures that directly address how collaborative resources are in their ability to exchange information (Schilling and Phelps, 2007), for example, Data Sharing (Information Transparency; Data Capture; Data Quality), Interoperability (Shared language; Knowledge Transfer; Common Tools), and Efficient IT Systems (Network Connectivity; Real-time Data Exchange; Efficient IT Infrastructure), in the case of multi-organisational networks (Harrington et al., 2012)

\subsection{Knowledge Mobility Mechanisms}

It is important to define various types of knowledge before designing the network, and selecting an appropriate mechanism to mobilise that knowledge. Building on sections 2.1-2.3., this section summarises the extant literature in the domain to inform the development of an integrated network-knowledge configuration framework.

\subsubsection{Knowledge types}

Objectivists understand knowledge as a static phenomenon, which can be managed as information, whereas, constructivists see knowledge as highly contextual - embedded either in a process, product or person (Kedia and Bhagat, 1988). As it is processed through a recreation process in the mind of the 'recipient' (El Sawy et al., 1998; Alavi and Leidner, 2001), it cannot be separated from its 'source' (Cook \& Brown, 1999). As knowledge is also linked to actions, knowledge is obtained through organisational tasks, in specific settings, which may be unique to the individual organisation (Dixon, 2000). Conversely, knowledge is also embedded in unique organisation processes, practices, norms and routines (Davenport and Prusak, 1998). The gained knowledge can be conceptualised, captured and transferred depending on its characteristics (Dixon, 1994; Sarker et al., 2005) and may provide the foundation for evaluating and incorporating new information and experiences, and in developing 'absorptive capacity' (Davenport and Prusak, 1998). Fosfuri and Tribo (2008) propose 4 stages in the development of such 'knowledge capacity'. It starts with the acquisition of [tacit] knowledge, followed by assimilation of the aforementioned knowledge (stage 2). The third stage involves the transformation of tacit knowledge, i.e. codification into explicit knowledge. Finally, competitive advantage is achieved by exploitation of this knowledge. For example, results show that the exchange of tacit and explicit knowledge in the context of a relationship between a supplier and a customer has a positive impact on the supplier's operational performance (Nagati and Rebolledo, 2013).

\subsubsection{Mechanisms for transfer}

In the context of this research, knowledge mobility mechanisms refer to the systems, methods, procedures or processes through which knowledge is transferred from the source to 
the recipient (Chai et al., 2003). Embedded in a complex, interdependent system, these mechanisms are dependent on the network configuration as well as the characteristics of the knowledge being shared. This is often dependent on multiple autonomous players with varying technical cultures (affecting knowledge mind-sets), managerial background (affecting decision knowledge) and supply chain management exposures (affecting knowledge sharing attitudes) (Wadhwa and Saxena, 2007) . Different transfer mechanisms will be more or less effective in different network configurations, and it will require different sharing mechanisms to transfer different types of knowledge.

In the literature, knowledge transfer has been defined as the identical or partial replication of knowledge from one place to another (Kostova, 1996; Szulanski, 1996), which can be replication (identical replication) or adaption (adapting existing knowledge to some degree). Transfer can also be intentionally structured (specific plan), accidentally unstructured (no framework) or diffuse (Berryman, 2005). Szulanski (1996), Dixon (2000), Gupta and Govindarajan (2000) and Berryman (2005) also focused on factors affecting knowledge mobility mechanisms. The following elements, described as dimensions of knowledge transfer, are regarded as having most impact in accelerating or inhibiting the outcome (ibid):

- Message (Knowledge): Content, Tacit - Explicit, Characteristics

- Source (Source of Knowledge): Profile, Attributes (Age, Size, capability), Motivation to share, Resource capacity

- Receiver (Recipient of Knowledge): Profile, Attributes (Age, Size, capability), Level of experience, Motivation, Effort, Absorptive capacity

- Channel (Organisational Context): Characteristics, Cultural differences, Organisational impact scope, Economic incentives, Environment factors, Broad task environment, Task frequency, KT experience, Activities and modes transferring knowledge.

These dimensions provide a basis for understanding the communication processes involved, by characterising the individual parameters affecting the process. As successful knowledge sharing is seen as including a process of learning interactions (Szulanski, 2003), further dimensions are considered here - in moving to a more complete model capturing the individual parameters as well as their relationships. In his comprehensive literature review on knowledge sharing, Cummings (2003) identified five primary contexts that can affect knowledge internationalisation, e.g. the relationship between the source and the recipient, the form of the knowledge, the recipients learning preposition, the knowledge-sharing capability of the source, and the broader environment of the knowledge sharing process. Furthermore a series of key factors were highlighting which affect the outcome of the sharing activities (ibid), namely:

- Environmental factors: focus on economic, cultural, political, industrial and institutional trends and drivers that influence relational, knowledge, source and recipient contexts (Allen 1977; Tushman 1977; Hedberg 1981; Sagafi-nejad 1990; Almeida and Grant 1998; Yeung et al. 1999; Kostova, 1999; Kim and Nelson 2000; Harrington and Srai 2012) 
- Relational factors: Organisational distance; Physical distance; Institutional distance; Knowledge competence; Relationship distance. Focus on partnerships dependent on structure, location, governance mechanism and expertise (Graham 1985; Hofstede 1980; Ouchi 1980)

- Recipient and Source factors: focus on the multi-organisational network partners participating in the knowledge sharing process - in terms of Motivation; Capability; Absorptive and learning capacity: Collaborative experience; Knowledge experience; Credibility; Retentive capacity; Learning culture (Cohen and Levinthal 1990; Doz 1996; Powell et al. 1996; Hamel 1991; Szulanski 1996; Dixon 2000; Simonin 1997; Prusak 1999)

- Knowledge factors: Explicitness; Embeddedness

Tacitness and embeddedness have mostly been referred to in the literature when reviewing knowledge characteristics and complexity (Doz and Santon 1997). It is well understood that it is easier to transfer explicit knowledge than tacit knowledge and - for this reason - it is desirable to convert tacit knowledge to explicit knowledge wherever possible (Fleet et al., 2014). In terms of definitions, Chai et al, (2003) summarised these dimensions as follows:

- Explicit Knowledge: Low tacitness and low embeddedness; Codified or verbalised and less context specific

- Experimental Knowledge: High tacitness and low embeddedness; Acquired through experience and practice

- Endemic Knowledge: Low tacitness and high embeddedness; Articulated but only meaningful when fully understood

- Existential Knowledge: High tacitness and high embeddedness; Acquired through experience and practice but only meaningful in a particular environment as it is less applicable outside this specific environment.

Lam $(1997,2000)$ also defined four types of knowledge, in relationship to their tacitness, but instead of looking at the embeddedness of the message, he analysed where the knowledge is held, if within an individual or a group of people. Chen and McQueen (2008) summarised the four types as follows and extended to capture insights about levels of knowledge experience (see figure 3):

- Embrained Knowledge: Individual and explicit; Dependent on the skills and abilities of an individual able to be articulated (e.g. Theoretical knowledge)

- Embodied Knowledge: Individual and tacit; dependent on individual experience and practice and is complex to transfer (e.g. practical experience)

- Encoded Knowledge: Collective and explicit; articulated, accumulated knowledge from groups of individuals (e.g. written procedures)

- Embedded Knowledge: Collective and tacit; Accumulated knowledge from groups of individuals which is embedded in organisations rules and procedures (e.g. routines) 


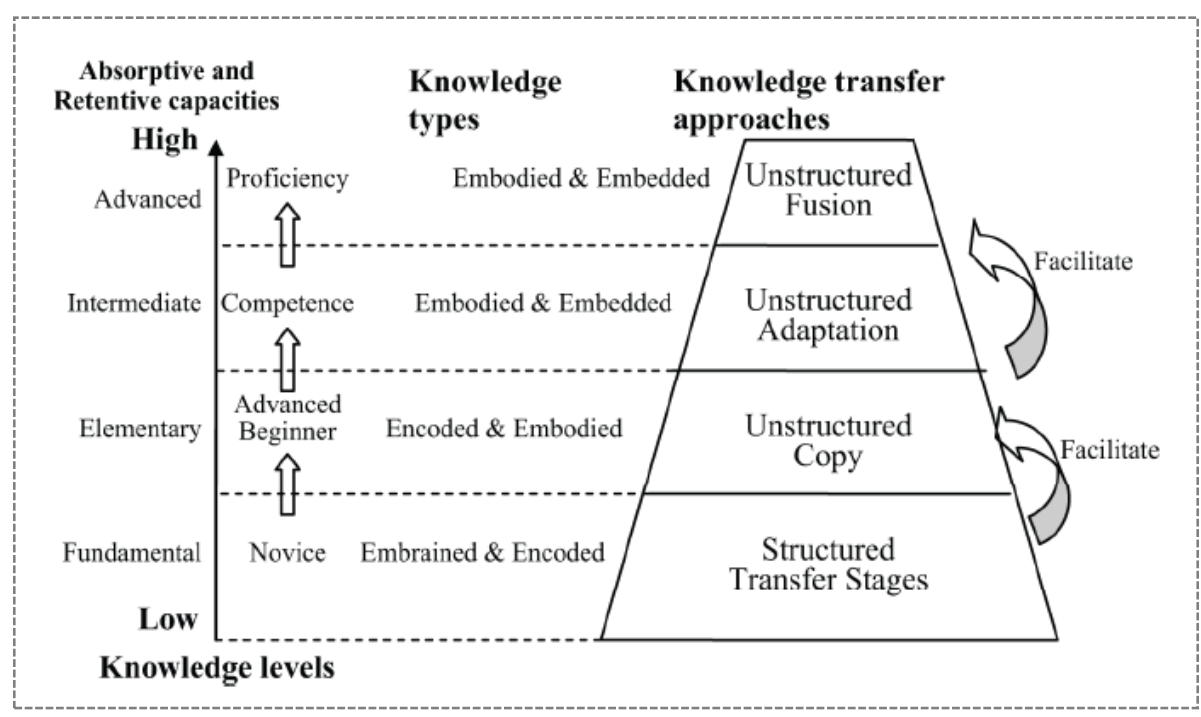

Figure 3: Knowledge Transfer Type Adoption Model (from Dreyfus and Dreyfus 1986, Chen and McQueen, 2008)

Chai et al. (2003) summarised knowledge sharing mechanisms identified in the management literature, as well as their characteristics within the dimensions of reach and richness. Table 1 summarises examples of knowledge transfer mechanisms (adapted from De Meyer 1991; Chiesa and Manzini 1996; Almeida and Grant 1998; Chai et al., 2003; Chen and McQueen, 2009) aligned with different types of knowledge, levels of knowledge, characteristics, and how they may affect KMob processes (barriers and opportunities), to inform development of the KMC framework. 
Table 1: Examples of Knowledge Transfer Mechanisms

\begin{tabular}{|c|c|c|c|c|}
\hline Mechanism & Definition & Advantage (+) Disadvantage (-) & Knowledge type & Example \\
\hline $\begin{array}{l}\text { Transfer of people: } \\
\text { expatriation }\end{array}$ & $\begin{array}{l}\text { Transferred source works in recipient site for } \\
\text { fixed period, allowing 'one-to-many' expert } \\
\text { interactions }\end{array}$ & $\begin{array}{l}\text { (+) One-to-many potential; high richness } \\
\text { (-) Limited to sources knowledge; low reach }\end{array}$ & Experiential & $\begin{array}{l}\text { Transfer: Technical know-how, how-why, } \\
\text { know-who (e.g. problem solving skills) }\end{array}$ \\
\hline $\begin{array}{l}\text { Transfer of people: } \\
\text { overseas training }\end{array}$ & $\begin{array}{l}\text { Recipient sent to lead site to learn knowledge } \\
\text { from multiple experts (many-to-one) }\end{array}$ & $\begin{array}{l}\text { (+) Different knowledge perspectives } \\
\text { (-) High knowledge loss risk }\end{array}$ & Existential & $\begin{array}{l}\text { Transfer: Technical know-how, how-why, } \\
\text { know-who (e.g. specific operating skills) }\end{array}$ \\
\hline $\begin{array}{l}\text { Forums: } \\
\text { Conferences, } \\
\text { meetings }\end{array}$ & $\begin{array}{l}\text { Periodic meeting (often annually) of technical } \\
\text { staff working in similar areas from different } \\
\text { locations }\end{array}$ & $\begin{array}{l}\text { (+) Creating informal employee networks; } \\
\text { creating awareness of knowledge } \\
\text { (-) Can be costly; Difficult to quantify gains }\end{array}$ & Endemic & $\begin{array}{l}\text { Awareness, Transfer: Technical knowledge } \\
\text { (e.g. specific set-up procedures) }\end{array}$ \\
\hline International teams & $\begin{array}{l}\text { Teams with members from different locations } \\
\text { engaged in improvement activities, working on a } \\
\text { specific project. }\end{array}$ & $\begin{array}{l}\text { (+) Stable structure for further practice } \\
\text { (e.g. development of playbooks by virtual } \\
\text { teams) } \\
\text { (-) Can be costly; Difficult to quantify gains }\end{array}$ & Endemic & $\begin{array}{l}\text { Awareness, Transfer: Innovations, } \\
\text { procedures, technology }\end{array}$ \\
\hline Boundary spanner & $\begin{array}{l}\text { Interfaces, improving technical knowledge } \\
\text { transfer; collecting and broadcasting information } \\
\text { (improvements, skills, knowledge) }\end{array}$ & $\begin{array}{l}\text { (+) Filtering and channelling } \\
\text { (-) Additional resources required }\end{array}$ & Explicit & $\begin{array}{l}\text { Awareness: No transfer, connecting source } \\
\text { and recipient }\end{array}$ \\
\hline Audit & Data collection exercise on location performance & $\begin{array}{l}\text { (+) Creates awareness; High reach } \\
\text { (-) Resource consuming; No tangible } \\
\text { return guaranteed }\end{array}$ & Explicit & $\begin{array}{l}\text { Awareness: Know-who, know-what (e.g. } \\
\text { FMEA) }\end{array}$ \\
\hline Benchmarking & $\begin{array}{l}\text { Location visits to learn about mechanisms and } \\
\text { improvements at other sites (e.g. annual forum) }\end{array}$ & $\begin{array}{l}\text { (+) Effective, wide application range } \\
\text { (-) Costly; time and resource consuming }\end{array}$ & Endemic & $\begin{array}{l}\text { Transfer: Know-how, know-why (e.g. specific } \\
\text { set-up procedures) }\end{array}$ \\
\hline $\begin{array}{l}\text { Best practice } \\
\text { guidelines; Standard } \\
\text { Operating } \\
\text { Procedures (SOP) }\end{array}$ & $\begin{array}{l}\text { Collection and dissemination of knowledge } \\
\text { through the transfer of identified best practices }\end{array}$ & $\begin{array}{l}\text { (+) Cost effective; High reach; Knowledge } \\
\text { loss protection } \\
\text { (-) Large effort required to keep up-to-date } \\
\text { (revision control); Potential of low richness } \\
\text { (lacking background insights and context) }\end{array}$ & $\begin{array}{l}\text { Explicit } \\
\text { Endemic }\end{array}$ & $\begin{array}{l}\text { Transfer: Process guides, step-by-step } \\
\text { troubleshooting guides, virtual team } \\
\text { playbooks SOPs, rules, manuals, standards } \\
\text { (e.g. Intel 'copy exactly' philosophy) }\end{array}$ \\
\hline Periodicals, reports & $\begin{array}{l}\text { Internal periodic publications (journal, } \\
\text { newsletter) with articles on technological } \\
\text { knowledge development }\end{array}$ & $\begin{array}{l}\text { (+) Cost effective; High Reach } \\
\text { (-) Information overload; Language issues }\end{array}$ & $\begin{array}{l}\text { Explicit } \\
\text { Endemic }\end{array}$ & $\begin{array}{l}\text { Awareness, Transfer: Technical know-how } \\
\text { 'what can be done', who's who (e.g. problem } \\
\text { solving methods, specific set-up procedures) }\end{array}$ \\
\hline Electronic linkages & $\begin{array}{l}\text { Intranet: stores and disseminates information } \\
\text { Groupware: Guidelines, plant performance, } \\
\text { meeting minutes, procedures, journals, } \\
\text { discussion forums, project schedules } \\
\text { Tele/video conferencing: overseas } \\
\text { communication } \\
\text { Email: Weekly reports, problem solving advice } \\
\text { guidelines } \\
\text { Fax: Technical drawings, blueprints } \\
\text { Chat: Problem solving (advice), networking, } \\
\text { discussion }\end{array}$ & $\begin{array}{l}\text { (+) Cost effective; High reach } \\
\text { (-) Information overload; Language issues }\end{array}$ & $\begin{array}{l}\text { Explicit } \\
\text { Endemic }\end{array}$ & $\begin{array}{l}\text { Awareness, Transfer: Know-who, know-what, } \\
\text { know-how, know-why (e.g. problem solving } \\
\text { methods, FMEA, specific set-up procedures) }\end{array}$ \\
\hline
\end{tabular}




\section{Prototype Framework Development}

Based on outputs from the literature review section in section 2, three dimensions of Knowledge Mobility Configuration (KMC) were defined as:

1. Network Configuration: The network in which the transfer takes place in, capturing the 'source' and 'recipient' as well as all other aspects of the network and their relationship

2. Knowledge Context: The message which is being transferred and its characteristics

3. Knowledge Mobility Mechanism: The transfer processes and their characteristics

This framing provides a basis to develop a broad perceptive of the individual dimensions of knowledge mobility - and their relationship to network configuration - in order to develop a framework to be further refined through application and test using a series of case studies ( see table 2). The different aspects of network configurations affecting knowledge mobility processes are captured as Structure, Network Dynamics, Governance and Coordination, Support Infrastructure, Relationships, and Maturity Level. Knowledge Mobility Configuration emerging dimensions of analysis, derived from the literature review, are presented in table 3. This approach allows for an overall structure for a KMC framework, providing a holistic view of all dimensions and their relationship. The following research questions have also been defined to inform this research study, namely:

(1) Network Context: What dimensions need to be captured and how are these utilised to ensure maximum effectiveness of the knowledge transfer?

(2) Knowledge Context: What knowledge mobility mechanisms should be used to transfer different types of knowledge?

(3) Knowledge Mobility Mechanism: What knowledge mobility mechanisms should be used to transfer the different types of knowledge, and how are these best employed to support different network configurations? 
Table 2: Knowledge Mobility Configuration - Prototype framework and dimensions of analysis

\begin{tabular}{|c|c|c|c|}
\hline \multicolumn{4}{|c|}{ Network Configuration } \\
\hline Network Phase & Nascent & Emerging & Mature \\
\hline $\begin{array}{l}\text { Supply Network } \\
\text { Stage }\end{array}$ & $\begin{array}{l}\text { Embryonic } \\
\text { Fragmented }\end{array}$ & $\begin{array}{l}\text { Formation } \\
\text { Expansion } \\
\text { Stabilisation }\end{array}$ & Established \\
\hline \multicolumn{4}{|l|}{ Dimensions } \\
\hline Structure & \multirow{5}{*}{\multicolumn{3}{|c|}{$\begin{array}{l}\text { Explore emerging dimensions influencing Knowledge Mobility processes } \\
\text { - Geographical footprint of a network, including the dispersion (shape, } \\
\text { levels of vertical and horizontal integration) of network units and their } \\
\text { interdependence (partnerships, ownership, flexibility) } \\
\text { - Strategic orientation on process, material and information flow } \\
\text { - Governance systems and mechanisms } \\
\text { - Infrastructure supporting process, material and information flow } \\
\text { - Maturity Levels: Emergence of product line and establishment of the } \\
\text { network } \\
\text { See Table } 3 \text { for additional details }\end{array}$}} \\
\hline Network Dynamics & & & \\
\hline $\begin{array}{l}\text { Governance and } \\
\text { Coordination }\end{array}$ & & & \\
\hline $\begin{array}{c}\text { Support } \\
\text { Infrastructure }\end{array}$ & & & \\
\hline Relationships & & & \\
\hline \multicolumn{4}{|c|}{ Knowledge Context } \\
\hline $\begin{array}{c}\text { Knowledge } \\
\text { Characteristics }\end{array}$ & $\begin{array}{l}\text { Fundamental } \\
\text { Knowledge }\end{array}$ & Moderate Knowledge & $\begin{array}{l}\text { Advanced } \\
\text { Knowledge }\end{array}$ \\
\hline Tacitness & Low & Low/High & High \\
\hline Embeddedness & Low & Low/High & High \\
\hline $\begin{array}{l}\text { Individual- } \\
\text { Collective }\end{array}$ & $\begin{array}{l}\text { Individual } \\
\text { Embrained }\end{array}$ & $\begin{array}{l}\text { Collective } \\
\text { Encoded }\end{array}$ & $\begin{array}{l}\text { Individual and } \\
\text { Collective } \\
\text { Embodied and } \\
\text { Embedded }\end{array}$ \\
\hline Type & Explicit & $\begin{array}{l}\text { Experimental } \\
\text { Endemic }\end{array}$ & Existential \\
\hline Examples & $\begin{array}{c}\text { Theoretical } \\
\text { knowledge, written } \\
\text { rules, procedures }\end{array}$ & $\begin{array}{l}\text { Practical experience, } \\
\text { written rules, } \\
\text { procedures }\end{array}$ & $\begin{array}{c}\text { Practical } \\
\text { experience, } \\
\text { routines, norms }\end{array}$ \\
\hline $\begin{array}{l}\text { Knowledge } \\
\text { Transfer } \\
\text { Mechanism }\end{array}$ & \multicolumn{3}{|c|}{$\begin{array}{l}\text { Explore mechanisms of transfer in terms of Awareness, Transfer. Level } \\
\text { etc. Mechanisms are dependent on the network configuration as well as } \\
\text { the characteristics of the knowledge being shared. Different transfer } \\
\text { mechanisms will be more or less effective in different network } \\
\text { configurations, and it will require different sharing mechanisms to } \\
\text { transfer different types of knowledge } \\
\text { - See Table } 1 \text { and section } 2 \text { for supporting details }\end{array}$} \\
\hline
\end{tabular}


Table 3. Prototype Knowledge Mobility Configuration framework - emerging criteria

\begin{tabular}{|c|c|c|c|}
\hline $\begin{array}{l}\text { Network } \\
\text { Configuration } \\
\text { Dimension }\end{array}$ & $\begin{array}{l}\text { Network } \\
\text { Configuration } \\
\text { Sub-dimension }\end{array}$ & Definition & Examples of contributing literature \\
\hline \multirow{7}{*}{ Structure } & Dispersion & $\begin{array}{l}\text { Shape of network with respect to } \\
\text { levels of knowledge integration }\end{array}$ & $\begin{array}{l}\text { Tushman, 1977; Granovetter, 1985; } \\
\text { Uzzi, 1996; Argote, 1999; Liu et al., } \\
\text { Harrington and Srai, } 2017\end{array}$ \\
\hline & Interdependence & $\begin{array}{l}\text { Self-sufficiency of subsidiaries, } \\
\text { based on relationship and flexibility }\end{array}$ & $\begin{array}{l}\text { Phene et al., 2005; Zhao and Luo, } \\
2005\end{array}$ \\
\hline & $\begin{array}{l}\text { Organisational } \\
\text { context }\end{array}$ & $\begin{array}{l}\text { Organisational structural } \\
\text { arrangements (e.g. joint ventures); } \\
\text { Institutionalisation or internalisation - } \\
\text { degree to which the recipient obtains } \\
\text { ownership of, commitment to and } \\
\text { satisfaction with the transferred } \\
\text { knowledge }\end{array}$ & $\begin{array}{l}\text { Granovetter, 1985; Argote, 1999; } \\
\text { Cummings, } 2003\end{array}$ \\
\hline & $\begin{array}{l}\text { Subsidiaries } \\
\text { location }\end{array}$ & Physical distance between locations & $\begin{array}{l}\text { Jacobs, 1969; Galbraith, 1990; } \\
\text { Davenport and Prusak, 1998; } \\
\text { Wheeler, } 2001\end{array}$ \\
\hline & $\begin{array}{l}\text { Knowledge } \\
\text { transfer network }\end{array}$ & $\begin{array}{l}\text { Range, members, roles and } \\
\text { responsibilities Dependence or } \\
\text { independence on the knowledge } \\
\text { and the knowing subject }\end{array}$ & $\begin{array}{l}\text { Dreyfus and Dreyfus, 1986; Doz and } \\
\text { Prahalad, 1991; Von Krogh and Roos, } \\
\text { 1995; Berryman, 2005; Harrington and } \\
\text { Srai, } 2016\end{array}$ \\
\hline & Source abilities & $\begin{array}{l}\text { Establishment in terms of reputation, } \\
\text { practice and motivation; capability to } \\
\text { make use of external knowledge }\end{array}$ & $\begin{array}{l}\text { Cohen and Levinthal, 1990; Hamel, } \\
\text { 1991; Szulanski, 1996; Dixon, } 2000 \\
\text { Zahra and George, 2002; }\end{array}$ \\
\hline & Recipient abilities & $\begin{array}{l}\text { Establishment in terms of } \\
\text { motivation, intention, practice and } \\
\text { developing capacity }\end{array}$ & $\begin{array}{l}\text { Bandura, 1986; Argyris, 1990; Hamel, } \\
\text { 1991; Yeung et al., 1999; Prusak, } \\
\text { 1999; Pfeffer and Sutton, 2000; Fosfuri } \\
\text { and Tribo, } 2008\end{array}$ \\
\hline \multirow{3}{*}{$\begin{array}{l}\text { Network } \\
\text { Dynamics }\end{array}$} & Standardisation & $\begin{array}{l}\text { Strategic orientation of } \\
\text { manufacturing processes and key } \\
\text { activities }\end{array}$ & $\begin{array}{l}\text { Peteraf and Shanley, 1997; Harrington } \\
\text { and Srai, } 2012\end{array}$ \\
\hline & Production line & $\begin{array}{l}\text { Production planning; Strategic } \\
\text { orientation and management of } \\
\text { manufacturing, material and } \\
\text { information flow }\end{array}$ & $\begin{array}{l}\text { Guinery and MacCarthy, 2009; } \\
\text { Harrington and Srai, } 2012\end{array}$ \\
\hline & Knowledge status & $\begin{array}{l}\text { Definition (individual or collective) } \\
\text { and value of knowledge and } \\
\text { knowledge sharing within the } \\
\text { organisation; management at key } \\
\text { interfaces }\end{array}$ & $\begin{array}{l}\text { Hedberg, 1981; Dixon, 1994; Nonaka } \\
\text { and Takeuchi, 1995; Kostova, 1999; } \\
\text { Alavi and Leidner, 2001; Guinery and } \\
\text { MacCarthy. } 2009\end{array}$ \\
\hline \multirow{4}{*}{$\begin{array}{l}\text { Governance } \\
\text { and } \\
\text { Coordination }\end{array}$} & $\begin{array}{l}\text { Commercial } \\
\text { control }\end{array}$ & $\begin{array}{l}\text { Governance and coordination } \\
\text { systems around commercial } \\
\text { activities }\end{array}$ & $\begin{array}{l}\text { Sagafi-nejad, 1990; Yeung et al., } \\
\text { 1999; Harrington and Srai, 2012; } \\
\text { Harrington and Srai, } 2017\end{array}$ \\
\hline & $\begin{array}{l}\text { Engineering } \\
\text { control }\end{array}$ & $\begin{array}{l}\text { Governance and coordination } \\
\text { systems around engineering } \\
\text { activities }\end{array}$ & $\begin{array}{l}\text { Andrews, 1971; Barney, 1991; } \\
\text { Harrington and Srai, 2012; Harrington } \\
\text { and Srai, } 2017\end{array}$ \\
\hline & $\begin{array}{l}\text { Performance } \\
\text { measures }\end{array}$ & $\begin{array}{l}\text { Variables determining success; } \\
\text { effectiveness of the knowledge } \\
\text { transfer and its institutionalisation } \\
\text { (embedding knowledge within } \\
\text { receiving organisation); "Stickiness" } \\
\text { - degree to which knowledge is lost } \\
\text { during transfer }\end{array}$ & $\begin{array}{l}\text { Barney, 1991; Cowan and Foray, } \\
\text { 1997; Lehr and Rice, 2002; Szulanski, } \\
\text { 2003; Harrington et al., } 2012\end{array}$ \\
\hline & $\begin{array}{l}\text { Economic } \\
\text { labour/IP } \\
\text { incentives }\end{array}$ & $\begin{array}{l}\text { Governance and coordination } \\
\text { systems impact }\end{array}$ & Baliga and Jaeger, 1984; Argote, 1999 \\
\hline \multirow{3}{*}{$\begin{array}{l}\text { Support } \\
\text { Infrastructure }\end{array}$} & $\begin{array}{l}\text { Engineering } \\
\text { systems and } \\
\text { manufacturing } \\
\text { capability }\end{array}$ & $\begin{array}{l}\text { Engineering systems supporting } \\
\text { manufacturing operations and } \\
\text { efficiencies }\end{array}$ & $\begin{array}{l}\text { Von Hippel, 1988; Appleyard, 1996; } \\
\text { Harrington and Srai, 2012; }\end{array}$ \\
\hline & $\begin{array}{l}\text { Engineering } \\
\text { resources and } \\
\text { people skills }\end{array}$ & $\begin{array}{l}\text { Engineering resources supporting } \\
\text { manufacturing operations and } \\
\text { efficiencies }\end{array}$ & $\begin{array}{l}\text { Hofstede, 1980; Graham, 1985; } \\
\text { Almeida and Kogut, 1999; Harrington } \\
\text { et al., } 2012\end{array}$ \\
\hline & $\begin{array}{l}\text { Knowledge } \\
\text { transfer systems }\end{array}$ & $\begin{array}{l}\text { Mechanism in place, and usage, } \\
\text { across the whole network }\end{array}$ & $\begin{array}{l}\text { Szulanski, 1996; Davenport et al., } \\
\text { 1996; Hansen et al., 1999; Lev, 2001; } \\
\text { Wadhwa and Saxena, 2007 }\end{array}$ \\
\hline
\end{tabular}




\begin{tabular}{|c|c|c|c|}
\hline & Culture & $\begin{array}{l}\text { Cultural establishment between } \\
\text { subsidiaries (country and } \\
\text { organisational culture) }\end{array}$ & $\begin{array}{l}\text { Schein, 1985; Hofstede, 1997, 2001; } \\
\text { Cullen, 2002; Harrington et al., } 2012\end{array}$ \\
\hline & Language & $\begin{array}{l}\text { Status on agreement on common } \\
\text { language }\end{array}$ & $\begin{array}{l}\text { Enright, 2000; Almeida and Phene, } \\
\text { 2004; Song et al., } 2013\end{array}$ \\
\hline \multirow{8}{*}{ Relationships } & $\begin{array}{l}\text { Partnership - } \\
\text { supplier }\end{array}$ & $\begin{array}{l}\text { Linkages between network } \\
\text { members, their relationship and } \\
\text { value sets }\end{array}$ & $\begin{array}{l}\text { Andrews, 1971; Barney, 1991; } \\
\text { Harrington et al., 2012; Harrington and } \\
\text { Srai, 2016; Delbufalo, } 2017\end{array}$ \\
\hline & $\begin{array}{l}\text { Partnership - } \\
\text { customer }\end{array}$ & $\begin{array}{l}\text { Linkages between multi- } \\
\text { organisational network members, } \\
\text { their relationship and value sets }\end{array}$ & $\begin{array}{l}\text { Allen, 1977; Tushman, 1977; Romero } \\
\text { and Molina, 2011; Harrington et al., } \\
\text { 2012; Dooley et al., 2013; Harrington } \\
\text { and Srai, } 2016\end{array}$ \\
\hline & $\begin{array}{l}\text { Intensity of } \\
\text { connection }\end{array}$ & $\begin{array}{l}\text { Inter-relations and time period of } \\
\text { previous connections }\end{array}$ & $\begin{array}{l}\text { Etzioni, 1961; Ouchi, 1980; Dixon, } \\
\text { 1994; Hansen, 1999; Bresman et., } \\
1999\end{array}$ \\
\hline & Contact frequency & $\begin{array}{l}\text { Frequency and purpose of contact } \\
\text { and how it is initiated }\end{array}$ & $\begin{array}{l}\text { Nonaka, 1994; Dixon, 1994; lansiti, } \\
\text { 1998; Yeung et al., } 1999\end{array}$ \\
\hline & Power distance & $\begin{array}{l}\text { Relationship between parties; } \\
\text { perceptions of inequality and } \\
\text { symmetry }\end{array}$ & $\begin{array}{l}\text { Allen, 1977; Almeida and Kogut, 1999; } \\
\text { Almeida and Phene, 2004; Phene et } \\
\text { al., 2005; Enright, 2000; Hofstede, } \\
\text { 2001; Song et al., } 2003\end{array}$ \\
\hline & Masculinity & $\begin{array}{l}\text { Willingness to promote societal } \\
\text { values }\end{array}$ & $\begin{array}{l}\text { Kedia and Bhagat, 1988; Zander and } \\
\text { Solvell, 2000; Gargiulo and Benassi, } \\
\text { 2000; Phene et al., 2005; Zhao and } \\
\text { Luo, } 2005\end{array}$ \\
\hline & Individualism & Degree of self interest & $\begin{array}{l}\text { Kedia and Bhagat, 1988; Triandis, } \\
\text { 1995; Hofstede, 2001; Gargiulo and } \\
\text { Benassi, } 2000\end{array}$ \\
\hline & $\begin{array}{l}\text { Uncertainty } \\
\text { avoidance }\end{array}$ & $\begin{array}{l}\text { Reluctance to deal with ambiguity } \\
\text { and lack of willingness to embrace } \\
\text { change }\end{array}$ & $\begin{array}{l}\text { Doz et al., 1981; Gupta and } \\
\text { Govindarajan, 1991; Kostova, 1996; } \\
\text { Hofstede, 1997; Almeida and Kogut, } \\
1999\end{array}$ \\
\hline Product & Configuration & $\begin{array}{l}\text { Production stage, product } \\
\text { differentiation and portfolio }\end{array}$ & $\begin{array}{l}\text { Srai and Gregory, 2008; Harrington } \\
\text { and Srai, } 2017\end{array}$ \\
\hline
\end{tabular}




\section{Research Methodology}

This section presents an overview of the research study' methodological approach. A mixed methodology was employed, involving expert panel input (interviews and benchmarking), followed by a multiple case study method. This multiple case study strategy is in line with Yin's definition (2003) of it being an empirical inquiry that investigates a contemporary phenomenon both in-depth and within its real-life context. The approach is particularly appropriate here as this study seeks to explore both practice-based (where the insights of key industrial stakeholders are critical) and emerging phenomena when research and theory may be at an exploratory or formative stage (Yin 2003; Hartley 2004). The overall research process is outlined in figure 4.

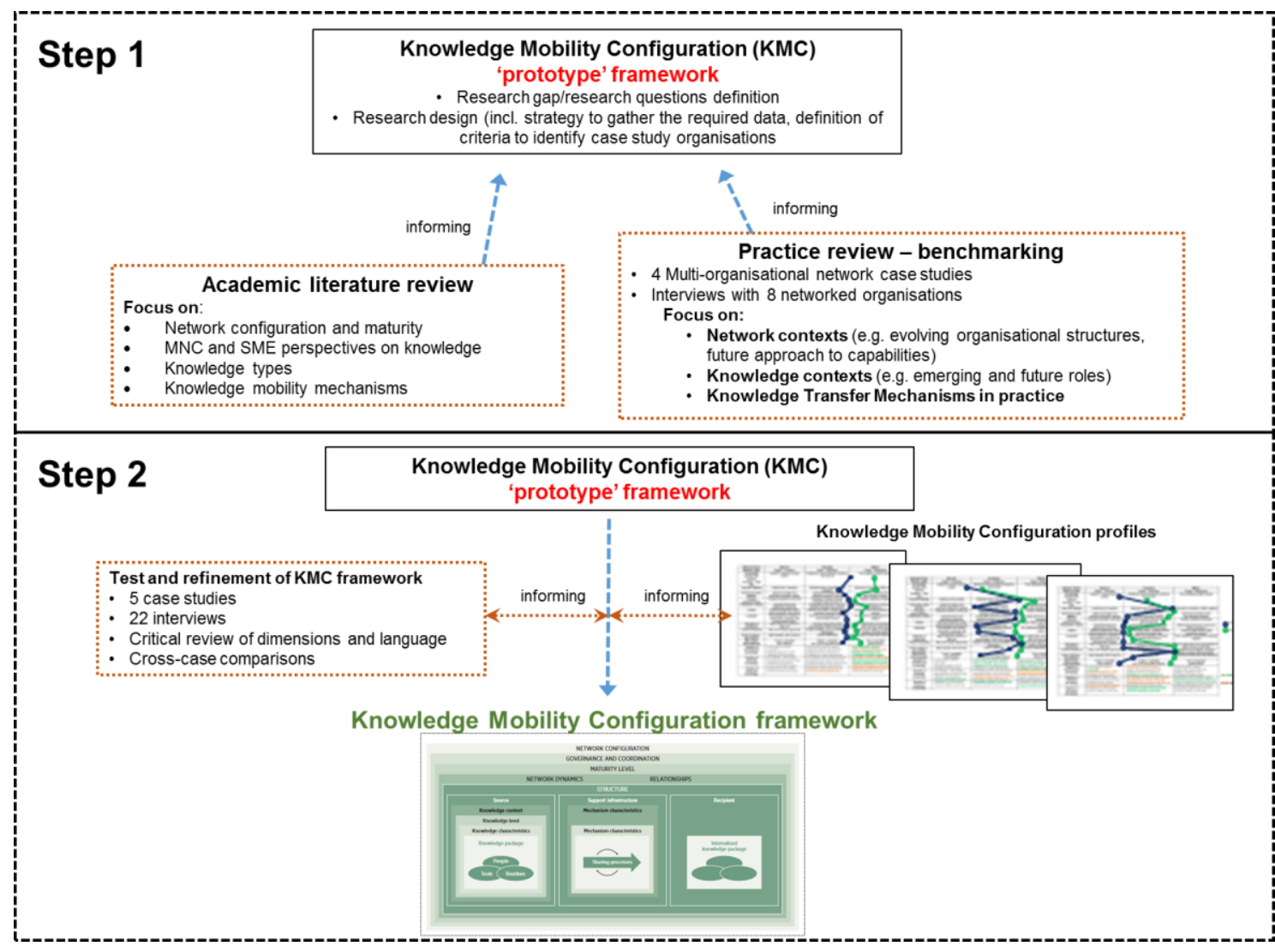

Figure 4: Research Design (adapted from Yin, 2003)

The first step, involved the design of an initial prototype framework, based on the literature review and discussions with industry experts. The research gap and associated research questions were defined, and an appropriate research design was established, highlighting the strategy to gather the required data, as well as defining criteria to enable organisations to be identified, and first contact established. This step also drew on findings from two benchmarking exercises: 
(1) Indicative of the growing shift towards information and knowledge-intensive activities involving multiple 'partners', a series of multi-organisational network cases were selected to examine different types of SME-SME and MNC-SME interactions. These spanned four industry sectors (Aerospace, Maritime, Energy, and Telecoms), took a network perspective, with multiple 'partners' involved in managing an increasing flow of knowledge and data, and activities across their geographically dispersed networks. The case studies were designed as semi-structured interviews and enabled dimensions from the literature review to be tested and, more importantly, brought into the industrial context. In summary:

- Two SMEs engaged in a dyadic collaboration, cooperating in an attempt to build better 'products' (Key insights across the cases included data availability and management, and communication plans; mechanisms to encourage trust and open-mindedness)

- Organisation historically acting as the more powerful party within a relationship (requiring large control over its supply chains and SMEs, thus creating dependency),

- Two firms selected from a legacy supply chain in order to investigate the complex nature of longer-term relationships and knowledge sharing (key insights across the cases centred on: intended reporting relationship to upper levels of management, and intended horizontal relationships with other units; conflict of interests, and resolution processes), and

- Case chosen due to a specific focus on aligning 'values' within an enterprise and extending these to its SME partners.

(2) Processes required to support both inter-firm and intra-firm knowledge network integration were explored through seven industrial case studies, and assessed in terms of evolving organisational structure, future approach to capabilities, and emerging and future roles:

- A review of models in current practice (e.g. capability and process models, enterprise architecture models, and 'lines of development'), roles and responsibility-type methodologies and activity definitions also examined where individual organisations and their knowledge networks had developed specific operational guidance, tactics, techniques and procedures, (Harrington and Srai 2016).

- Theoretical inputs from the academic literature that further informed knowledge design criteria, e.g. networked organisation (Zhang, Gregory, and Neely 2016), matrix structures (Kuprenas 2003), roles, responsibilities and disposition (Goold and Campbell 2003), and the emergence of more distributed systems (Srai et al., 2016). 
The second stage involved examination of the prototype framework dimensions and definitions using five case studies. Table 4 summarises the case organisations involved in this study, and the characteristics of their knowledge network under investigation. Selection criteria for the case studies, derived from step 1, included:

- 'Lead' location and case study location were located in different countries and are part of a wider global production network

- The case studies ideally would involve three product lines - with a 'lead' location in the EU or US, at least one production plant in the EU and/or US, with further locations in Asia as part of a global manufacturing network

- Knowledge and knowledge transfer activities were highly regarded and evidence that knowledge management mechanisms had been in operation within the organisation for at least five years.

- Access to different products with similar initial setup (type of product, 'lead' location in the same region) were available

- Availability of access to SMEs linked to MNC network and product type

For the five cases (MNC and SME), all product lines serve a similar application area, with the different case study products and their supply networks at different maturity levels. 15 senior engineers, three managers as well as four directors across MNC and SME case studies were interviewed (22 respondents in total).

Table 4: List of Case Organisations

\begin{tabular}{|c|c|c|c|c|c|}
\hline Case & $\begin{array}{l}\text { Lead } \\
\text { Location } \\
\text { Established }\end{array}$ & $\begin{array}{l}\text { 'Server' } \\
\text { Location }\end{array}$ & $\begin{array}{l}\text { Number of } \\
\text { Production } \\
\text { lines }\end{array}$ & $\begin{array}{l}\text { Network } \\
\text { Locations }\end{array}$ & $\begin{array}{l}\text { Classification } \\
\text { (Product/Production } \\
\text { line/supply network) }\end{array}$ \\
\hline $\begin{array}{l}\text { Network } \\
\text { A }\end{array}$ & 2001 & 2001 & 10 & $\begin{array}{l}4 \text { (EU, US, } \\
\text { Asia x 2) }\end{array}$ & Mature/Mature/Mature \\
\hline $\begin{array}{l}\text { Network } \\
\text { B }\end{array}$ & 2005 & 2012 & 13 & $\begin{array}{l}5(E U \times 2 ; \\
\text { US, Asia } \times \\
\text { 2) }\end{array}$ & $\begin{array}{l}\text { Established/Emerging } \\
\text { /Emerging }\end{array}$ \\
\hline $\begin{array}{l}\text { Network } \\
\text { C }\end{array}$ & 2006 & 2013 & 4 & $\begin{array}{l}4(E U, U S \\
\text { Asia } \times 2)\end{array}$ & $\begin{array}{l}\text { Early } \\
\text { Established/Emerging } \\
\text { /Nascent }\end{array}$ \\
\hline $\begin{array}{l}\text { Network } \\
\text { D }\end{array}$ & 2010 & 2013 & 1 & 1 (UK) & $\begin{array}{l}\text { Emerging/Emerging } \\
\text { /Nascent }\end{array}$ \\
\hline $\begin{array}{l}\text { Network } \\
\text { E }\end{array}$ & 2010 & 2013 & 1 & $\begin{array}{l}3 \text { (UK; EU; } \\
\text { Asia) }\end{array}$ & $\begin{array}{l}\text { Nascent/Nascent } \\
\text { /Nascent }\end{array}$ \\
\hline
\end{tabular}

The purpose of the interviews included, not only mapping of the different cases using the $\mathrm{KMC}$ framework, but also the refinement of the framework. The process explored the research questions set out in section 3 , and gathered the following information:

1. Background information on the organisation and its $\mathrm{KMob}$ activities

2. Background information on the product and supply network (local v. regional v. global perspectives) 
3. Current state Knowledge Mobility Configuration Profile - individual perceptions

4. Collective feedback on the different dimensions and their classifications

5. Overview of the different KT Mechanism applied and their application range

6. Perception on the ideal state for the current and future state of the knowledge mobility configuration

\section{Knowledge Mobility Configuration Profiling}

The case studies selected provide this study with examples of MNCs and SMEs collaborating in an emerging industry context, where novel end products are being manufactured using a hybrid of traditional techniques with emerging technologies. It is comprised of new entrants driving innovative manufacturing processes, coupled with more established firms looking to diversify their product portfolios. This section summarises application and test of the prototype framework. Limitations of space constrain the full graphical presentation for each Knowledge Configuration Profile generated through testing the prototype framework Figures 5, 6 and 7set out partial profiles for three of the cases (A, B, and $C$ ). In the summary of the findings (section 6.2 ), tables $6-8$ provide a summary of a comparative analysis involving all five cases

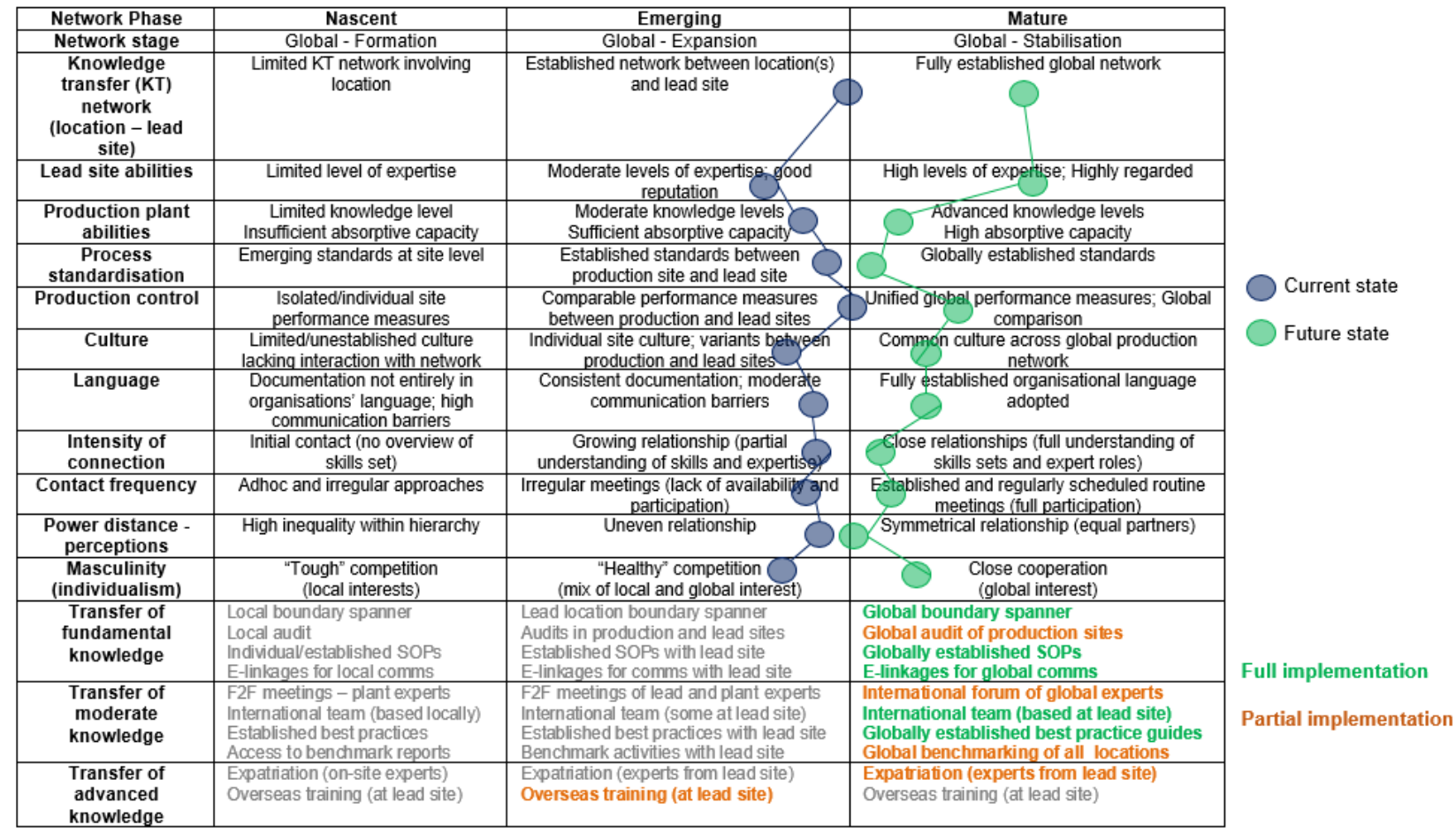

Figure 5. Knowledge mobility network configuration profile A - MNC (US-Global)

Profile A: Case A has been operational for many years and displays many of the characteristics of a mature knowledge network. However, while management and engineers agreed on many points, gaps were identified where the perceptions of management and 
engineers differed in terms of improving their 'knowledge sharing' network, and in bringing their product line closer to 'maturity' in terms of absorptive capacity. Owing to the maturity of the product line, it is unsurprising that there are many global knowledge mobility mechanisms in use today. Interestingly, some are less implemented at this location given that there is significant local knowledge. This has resulted in elements of certain sharing mechanisms deemed surplus to requirements. This was recognised as being high risk to certain large product lines as the knowledge share of vital information could be slowed by an assumed knowledge from locations, thus leading to isolation.

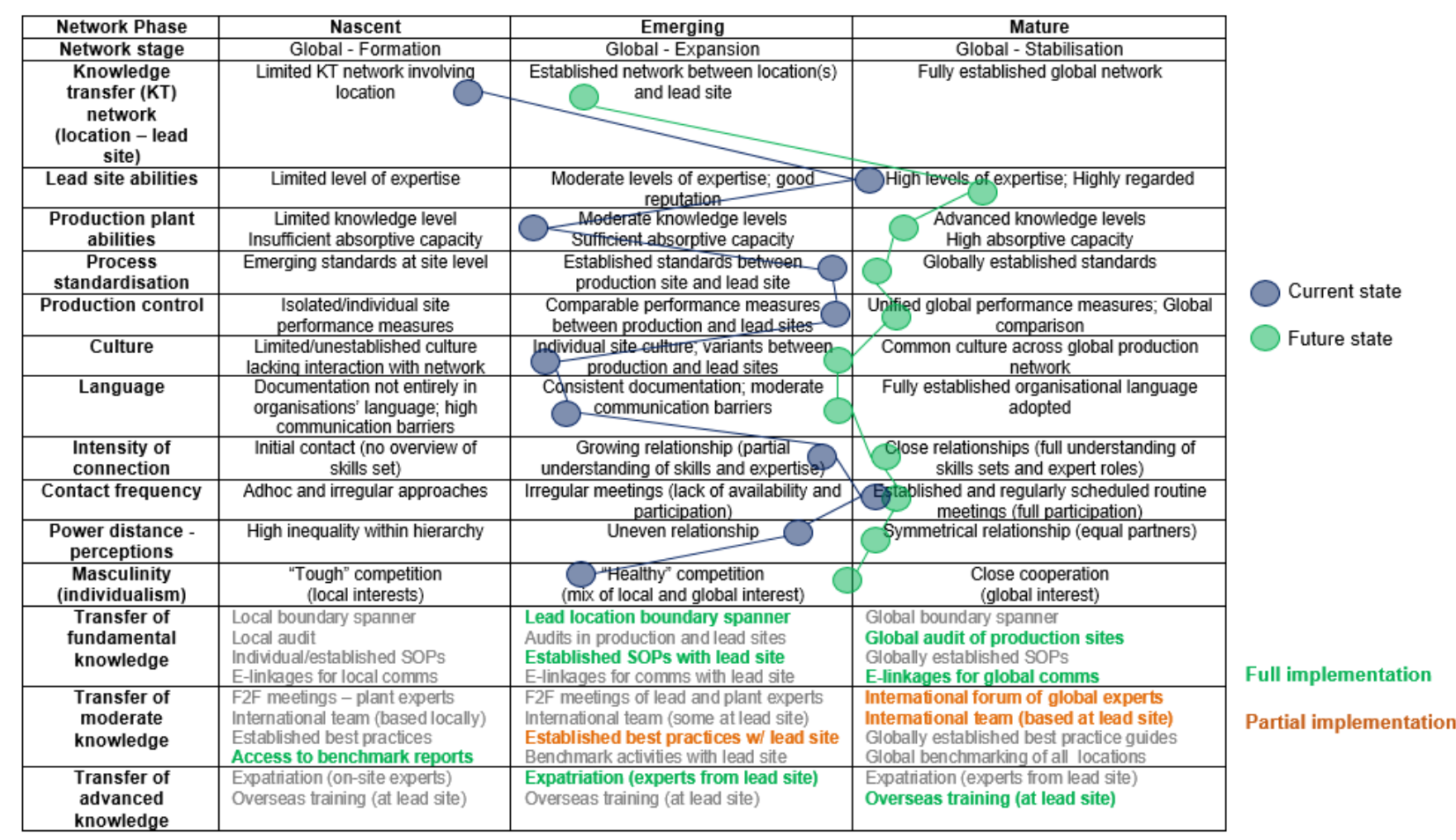

Figure 6. Knowledge mobility network configuration profile B - MNC (US-Regional)

Profile B: Case B's product line has been in global production for several years, with an expectation that the location (and its local suppliers, including SMEs) should play a leading role in future global product line development, scale-up, and transfer. This is reflected by the high expectations dictated by management in the knowledge configuration framework with gaps expected to close once more confidence and interaction between locations and network partners intensifies. Case B presents a typical illustration of a new product line within a global network context, involving the focal firm and its SME partners. The variation of knowledge mechanism use reflects the different stages that the engineers and managers are experiencing and operating in, indicating that these mechanisms greatly influence the specific product line and type of knowledge being transferred between partners, and vice-versa. Two 
main areas of concern are the lead location supplying insufficient knowledge, and language difficulties when interpreting original documentation.

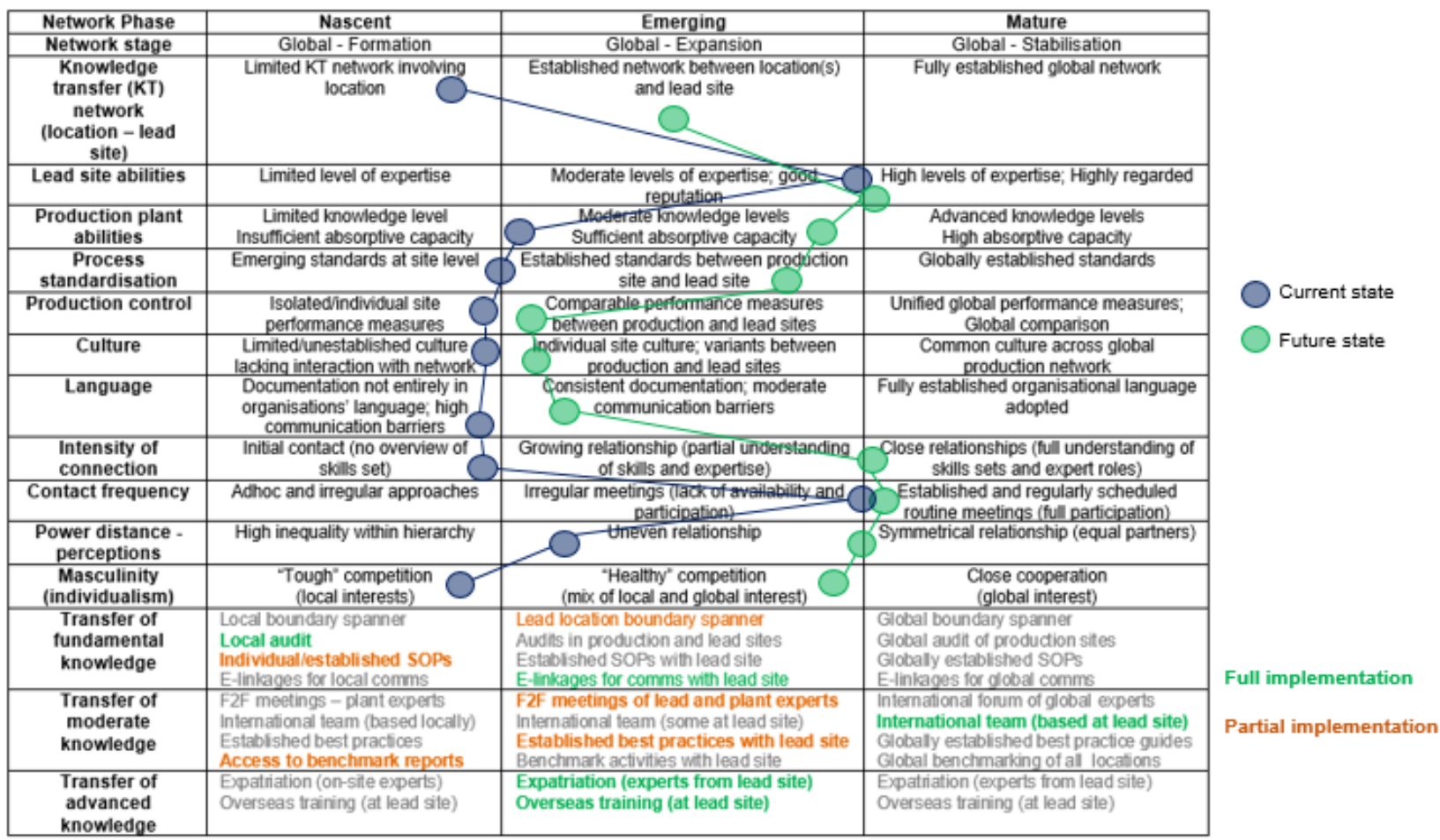

Figure 7. Knowledge mobility network configuration profile C - MNC (US-EU)

Profile C: Case C involves a production line, which is in its early stages of development and transfer, with a team heavily concentrated on gathering as much knowledge from the lead location as possible. In addition the specific line is currently being rolled out to multiple locations, which some at further stages of development than others, with more heavily reliant on a local SME supplier base. The lead location is also finding it difficult to allocate sufficient resources to assist this location. As this production line is in the early stages of implementation, there is little surprise that knowledge mechanisms have a large influence on the team. The main focus is on information at this stage, but this is due to change when other locations begin to come on-line, requiring more sharing of knowledge on their experiences.

Profile D: Case D is a developer and manufacturer, established in 2010. Benefiting from its high-speed manufacturing capability and low fabrication costs, the SME has seen its network dispersion grow from a single location to have a global reach in less than 12 months. In addition to increased dispersion, the strength of its partnerships has also increased and stabilised. It is focused on growing actors for development and production, who cover the entire value chain and complementary sectors. Future focus areas for the SME, identified using the framework, is the assessment of innovation and education activities that have a 
structured mentoring and networking programme, for example, leveraging their MNC networks to gain professional training combined with hands-on experience, engaging in SPOCs (Specialized Private Online Courses) tailored to the specific demands of the SME, and using 'rotating elevator' workshops with network partners for knowledge sharing.

Profile E: Case E is an SME whose specialised capability lies in ultra-thin and low-cost flexible microcircuits that may be incorporated into mass-market objects and packaging. With a well-developed regional customer-base in the UK, it is becoming increasingly affected by global customer pull, with no mature supply network structure to support this opportunity. Case $E$ is heavily focused on managing internal capabilities at present, where many issues of knowledge integration may be dealt with relatively simply, and even informally. Here, new actors and enterprises fail to adequately coordinate the external resource capabilities, core non-technical activities, and knowledge they will require in growing a business. However, in developing a longer-term network strategy view there is growing awareness of established networks for which case $E$ could leverage in terms of knowledge and capability. One of the main challenges SMEs face is affordable access to targeted and meaningful education and training in their particular field. Future focus areas for the SME, identified using the framework, included knowledge management, social media, and virtual teams linked to Knowledge Innovation Communities (KICs) and the emerging assets commonly utilised in this space - Massive Open Online Courses (MOOCs) and SPOCs (Specialized Private Online Courses), and Moodle as a flexible Virtual Learning and knowledge exchange tools.

\section{Summary of findings}

Previous studies have largely focused on large, well-established, market driven MNCs, their established products and their extended networks. In terms of theoretical contribution, this study informs knowledge mobility mechanisms for both networked SMEs and MNCs, which currently provide only limited detail on configuration elements and options on engagement with their extended networks. The research presents instructive results from case studies on the application of knowledge management approaches based on real world applications, and is organised in two sub-sections. Section 6.1 summarises the key findings from the benchmarking exercises. Section 6.2 provides a summary of the comparative analysis involves the five cases and then sets out the final KMC framework. 


\subsection{Benchmarking}

This section summarises outputs from the benchmarking studies, in terms of insights involving multi-organisational networks involved in knowledge-intensive activities (6.1.1), and operational practice inputs involving future knowledge network configurations (6.1.2).

\subsubsection{Insights on factors affecting future Multi-Organisational Networks}

Findings suggest that defining and aligning value sets of MNCs and SMEs operating within collaborative networks is crucial as it represents a means of assessing effective knowledge network integration and operational objectives. A number of dimensions have been identified. The importance of a series of dimensions that define value sets (and by extension knowledge) within a network context varied depending on both the nature of the service contract and partnering agreement (for example some networks identified cooperation, trust, commitment to objectives, commonality of objectives, defined roles, responsiveness to partners/problems, communication and equal rewards to be key, other networks valued respect of IP, and data security). In summary:

- Key insights across SME-SME cases engaged in dyadic collaborations included data availability and management, and communication plans; mechanisms to encourage trust and open-mindedness

- Key insights across MNC-SME cases involving longer-term relationships and knowledge sharing centred on: intended reporting relationship to upper levels of management, and intended horizontal relationships with other units; conflict of interests, and resolution processes)

\subsubsection{Insights on factors affecting future Knowledge Mobility Configurations}

Drawing on dimensions of network configuration from section 3, industrial context, potential network configuration options and stages, and the processes required to support both interfirm and intra-firm knowledge network integration were explored through seven industrial case studies. Table 5 summarises insights on key factors affecting future knowledge mobility configurations, in terms of:

- Evolving Organisational Structure

- Future approach to Capabilities

- Emerging and Future Roles

In summary, organisations with similar configuration characteristics, as currently set up, are looking to improve project delivery and grow new capabilities by increasingly moving towards partnering with other organisations (SMEs and MNCs), which is resulting in new roles emerging. Those organisations also seeking to adopt a similar future configuration have the 
following features of improved networking to offset skills imbalances, and transferring staff into other functions to broaden skills and knowledge of the wider business. As part of the 'futuring' process, new learning and career structures will be needed to encourage mobility, which may pose a significant challenge as resources may be reluctant to move across boundaries, in some cases. Moving to a virtual team model is a change management process and needs to be managed as such. Hence, cultural change is encouraged to develop a more flexible skill set and knowledge base, and at an organisational level, recognition that what is best for a 'virtual team', may not be best for a particular location. 
Table 5. Benchmarking

\begin{tabular}{|c|c|c|c|c|c|c|c|}
\hline $\begin{array}{l}\text { Insights on } \\
\text { 'future' } \\
\text { Knowledge } \\
\text { Mobility } \\
\text { Configuration }\end{array}$ & Organisation A & Organisation B & Organisation C & Organisation D & Organisation E & Organisation $\mathbf{F}$ & Organisation G \\
\hline $\begin{array}{l}\text { Evolving } \\
\text { Organisational } \\
\text { Structure }\end{array}$ & $\begin{array}{l}\text { Configuration } \\
\text { heavily driven by } \\
\text { partnering, drivers } \\
\text { recognised as } \\
\text { being resource- } \\
\text { people-location- } \\
\text { cost, with cost not } \\
\text { the major driver. } \\
\text { Responsiveness } \\
\text { to the market and } \\
\text { dynamics seen as } \\
\text { critical. }\end{array}$ & $\begin{array}{l}\text { Configuration heavily } \\
\text { driven by Global } \\
\text { network. } \\
\text { Location driven by } \\
\text { closeness to } \\
\text { customer. } \\
\text { Speed to market } \\
\text { critical (de-centralised) } \\
\text { viewed as more } \\
\text { important than cost } \\
\text { savings (centralised). } \\
\text { Use of Competency } \\
\text { Centre-type concepts } \\
\text { popular with Strategy } \\
\text { and/or Global Teams } \\
\text { linking these global } \\
\text { networks. }\end{array}$ & $\begin{array}{l}\text { Governance } \\
\text { largely central. } \\
\text { Location driven by } \\
\text { closeness to } \\
\text { manufacturing } \\
\text { sites with } \\
\text { development of a } \\
\text { global mind-set } \\
\text { e.g. global } \\
\text { communities of } \\
\text { practice. }\end{array}$ & $\begin{array}{l}\text { Configuration } \\
\text { heavily driven by } \\
\text { skills mismatch. } \\
\text { Key issue is the } \\
\text { potential surplus of } \\
\text { engineering skills in } \\
\text { traditional markets } \\
\text { versus.a shortage of } \\
\text { qualified } \\
\text { experienced people } \\
\text { in new markets. }\end{array}$ & $\begin{array}{l}\text { Competitiveness } \\
\text { seen as being driven } \\
\text { by network } \\
\text { configuration } \\
\text { dimensions } \\
\text { identified e.g. } \\
\text { Structure, Network } \\
\text { dynamics, } \\
\text { Governance and } \\
\text { coordination, } \\
\text { Support } \\
\text { infrastructure, and } \\
\text { Relationships } \\
\text { between network } \\
\text { members including } \\
\text { customers, suppliers } \\
\text { and users }\end{array}$ & $\begin{array}{l}\text { New approach to } \\
\text { Configuration with less } \\
\text { contact/lower synergies } \\
\text { between R\&D and } \\
\text { Manufacturing. } \\
\text { Stronger links between } \\
\text { R\&D, Marketing \& other } \\
\text { technology groups }\end{array}$ & $\begin{array}{l}\text { Configuration model based on shift } \\
\text { of selected 'engineering' activities } \\
\text { to low cost locations. } \\
\text { Given IP concerns outsourcing } \\
\text { unlikely to feature. } \\
\text { Future need for increased } \\
\text { servitization will change the nature } \\
\text { of operations (from OEM to a } \\
\text { greater service footprint) }\end{array}$ \\
\hline $\begin{array}{l}\text { Future approach } \\
\text { to Capabilities }\end{array}$ & $\begin{array}{l}\text { Approach to } \\
\text { capability is } \\
\text { leading to global, } \\
\text { virtual and } \\
\text { functional } \\
\text { engineering } \\
\text { communities of } \\
\text { practice with } \\
\text { greater emphasis } \\
\text { on responsibility } \\
\text { allocation. }\end{array}$ & $\begin{array}{l}\text { Critical to benefit from } \\
\text { expertise in various } \\
\text { regions. } \\
\text { R\&D also working } \\
\text { more extensively with } \\
\text { the rest of the } \\
\text { business - All IT } \\
\text { enabled. }\end{array}$ & $\begin{array}{l}\text { Internal partnering } \\
\text { considerable with } \\
\text { focus on adapting } \\
\text { internal processes }\end{array}$ & $\begin{array}{l}\text { Location skills used } \\
\text { as a source of global } \\
\text { capability. } \\
\text { Multiple sites } \\
\text { supported by central } \\
\text { engineering function } \\
\text { with best practice } \\
\text { transfer using a } \\
\text { Centre of Excellence } \\
\text { (CoE) model. }\end{array}$ & $\begin{array}{l}\text { Established } \\
\text { capabilities and } \\
\text { capability targets are } \\
\text { based on target } \\
\text { locations. } \\
\text { Greater focus on } \\
\text { relationships, } \\
\text { individuals and } \\
\text { working towards } \\
\text { more strategic long- } \\
\text { term partnerships. }\end{array}$ & $\begin{array}{l}\text { In terms of capability, } \\
\text { moving from internal } \\
\text { competencies towards } \\
\text { e.g. 'Intelligent } \\
\text { purchasers' - } \\
\text { Individuals/teams with } \\
\text { ability to understand the } \\
\text { technology and work } \\
\text { closely with partners. }\end{array}$ & $\begin{array}{l}\text { Capability focus - gaining } \\
\text { familiarity with common systems } \\
\text { given a need to increase } \\
\text { engineering knowledge in areas } \\
\text { outside of specific specialisms } \\
\text { (move to services). } \\
\text { Continuation of engineering teams } \\
\text { working with external } \\
\text { parties/different cultures. }\end{array}$ \\
\hline $\begin{array}{l}\text { Emerging and } \\
\text { Future Roles }\end{array}$ & $\begin{array}{l}\text { New roles of } \\
\text { functional } \\
\text { specialists capable } \\
\text { of networking } \\
\text { across an } \\
\text { organisation } \\
\text { emerging }\end{array}$ & $\begin{array}{l}\text { Large focus on people } \\
\text { working in } \\
\text { partnerships }\end{array}$ & $\begin{array}{l}\text { New roles } \\
\text { emerging } \\
\text { particularly related } \\
\text { to co-ordination, } \\
\text { reflected in } \\
\text { growing } \\
\text { importance of } \\
\text { softer skills }\end{array}$ & $\begin{array}{l}\text { Engineer transfer to } \\
\text { other functions to do } \\
\text { different role (e.g. } \\
\text { services), a } \\
\text { favoured approach }\end{array}$ & $\begin{array}{l}\text { Cross functional } \\
\text { teams/roles in } \\
\text { operation led by } \\
\text { Engineering e.g. } \\
\text { Transformation } \\
\text { project team/product } \\
\text { delivery team. }\end{array}$ & $\begin{array}{l}\text { Engineering roles - shift } \\
\text { from 'technical } \\
\text { specialist' to 'partner } \\
\text { working ', need for } \\
\text { people with both } \\
\text { technology and } \\
\text { relationship skills. "More } \\
\text { EQ (Emotional quotient) } \\
\text { than IQ". }\end{array}$ & $\begin{array}{l}\text { Engineering will continue to be } \\
\text { project driven: with added need for } \\
\text { an appreciation of other disciplines } \\
\text { and ability to handle customer } \\
\text { interface. No new positions } \\
\text { beyond those of e.g. product } \\
\text { lifecycle management }\end{array}$ \\
\hline
\end{tabular}




\subsection{Comparative analysis based on application of the KMC framework}

Although there are many ways an organization can acquire knowledge, there is broad consensus in the literature that organisational learning is a problem-solving process triggered by gaps between actual and potential performance (Pisano 1994). In terms of knowledge networks, there is often a wide disparity here, with SMEs focusing their knowledge activities on resolving technical issues, developing prototypes and securing investment. On the other hand, MNCs look to leverage existing capabilities, processes and techniques developed within their core industry. Tables 6-8 provide a summary of a comparative analysis involving all five cases, in terms of:

- Supply Network Configuration Profile - conventional dimensions of analysis

- Knowledge Mobility Configuration Profile - emerging dimensions of analysis

- Knowledge Mobility Configuration Profile - Knowledge Transfer Mechanisms 
Table 6. Supply Network Configuration Profile - conventional dimensions of analysis (extant theory)

\begin{tabular}{|c|c|c|c|c|c|}
\hline Network Type & $\begin{array}{l}\text { MNC (US-Global) } \\
\text { - Product Assembler }\end{array}$ & $\begin{array}{l}\text { MNC (US-Regional) } \\
\text { - Product Assembler }\end{array}$ & $\begin{array}{l}\text { MNC (US-EU) } \\
\text { - Product Assembler }\end{array}$ & $\begin{array}{l}\text { SME (UK) - Product } \\
\text { Assembler }\end{array}$ & $\begin{array}{l}\text { SME (UK) - Component } \\
\text { Manufacturer }\end{array}$ \\
\hline Network Phase & Mature & Emerging & Nascent & Emerging & Nascent \\
\hline Network Stage & Established & Expansion -> Stabilisation & Formation -> Expansion & Fragmented -> Formation & Embryonic \\
\hline $\begin{array}{l}\text { Structure } \\
\text { Dispersion } \\
\text { Interdependence }\end{array}$ & $\begin{array}{l}\text { Fully integrated (intra- } \\
\text { firm), perception of unity; } \\
\text { Independent and } \\
\text { autonomous }\end{array}$ & $\begin{array}{l}\text { Superficial intra-firm } \\
\text { integration (Perception of } \\
\text { a contractor relationship); } \\
\text { Partially dependent } \\
\text { (shared responsibilities) }\end{array}$ & $\begin{array}{l}\text { Defined inter-firm } \\
\text { relationship (Joint } \\
\text { Venture); Dependent } \\
\text { ('Lead' location remains } \\
\text { 'responsibility' owner) }\end{array}$ & $\begin{array}{l}\text { Fast growing diversity; } \\
\text { Increasing, flexible } \\
\text { linkages }\end{array}$ & Little structure, co-location \\
\hline $\begin{array}{l}\text { Network Dynamics } \\
\text { Standardisation }\end{array}$ & $\begin{array}{l}\text { Globally established } \\
\text { standards }\end{array}$ & $\begin{array}{l}\text { Established standards } \\
\text { between location and } \\
\text { 'lead' location }\end{array}$ & $\begin{array}{l}\text { Emerging standards at } \\
\text { 'location' level }\end{array}$ & $\begin{array}{l}\text { Fragmented with some } \\
\text { customisation }\end{array}$ & $\begin{array}{l}\text { Hybrid of customised and } \\
\text { established processes }\end{array}$ \\
\hline $\begin{array}{l}\text { Governance and } \\
\text { Coordination } \\
\text { Commercial Control } \\
\text { Engineering Control }\end{array}$ & $\begin{array}{l}\text { Control at the production } \\
\text { location level ('internal') }\end{array}$ & $\begin{array}{l}\text { Control at the 'lead' } \\
\text { location level ('external') }\end{array}$ & $\begin{array}{l}\text { Control at the organisation } \\
\text { level (centralised) }\end{array}$ & $\begin{array}{l}\text { VC Investment; Critical } \\
\text { capability driven; } \\
\text { Technology-driven }\end{array}$ & $\begin{array}{l}\text { Growing customer pull; Critica } \\
\text { capability driven }\end{array}$ \\
\hline $\begin{array}{l}\text { Support Infrastructure } \\
\text { Engineering Systems } \\
\text { Engineering Resources } \\
\text { Culture }\end{array}$ & $\begin{array}{l}\text { Identical systems across } \\
\text { the production network, } \\
\text { based on 'lead' location } \\
\text { specifications; Common } \\
\text { culture across global } \\
\text { production network }\end{array}$ & $\begin{array}{l}\text { Adjustment and } \\
\text { modification of lead } \\
\text { location systems (format } \\
\text { of specific documents); } \\
\text { Individual site culture, } \\
\text { variations between 'lead' } \\
\text { and locations }\end{array}$ & $\begin{array}{l}\text { Bespoke plant systems } \\
\text { (due to different } \\
\text { measurement units); No } \\
\text { established culture, } \\
\text { lacking interactions with } \\
\text { other parties }\end{array}$ & $\begin{array}{l}\text { Bespoke systems/tools; } \\
\text { Critical resources scale- } \\
\text { up; Trust moving to } \\
\text { commercial proposition }\end{array}$ & $\begin{array}{l}\text { Inter-system translation, small } \\
\text { scale production; Critical } \\
\text { resources only; } \\
\text { Trust-based }\end{array}$ \\
\hline $\begin{array}{l}\text { Relationships } \\
\text { Partnership-supplier } \\
\text { Partnership-customer }\end{array}$ & $\begin{array}{l}\text { Partnerships different } \\
\text { depending on location, } \\
\text { new and established with } \\
\text { local suppliers/customers }\end{array}$ & $\begin{array}{l}\text { Mix of partnerships as per } \\
\text { lead site, and new local } \\
\text { suppliers/customers }\end{array}$ & $\begin{array}{l}\text { Same partnerships as per } \\
\text { 'lead' location }\end{array}$ & $\begin{array}{l}\text { Partner selection; First } \\
\text { wave customer partnering }\end{array}$ & $\begin{array}{l}\text { Partner selection; Project } \\
\text { specific/ group partnerships }\end{array}$ \\
\hline
\end{tabular}


Table 7. Knowledge Mobility Configuration Profile - emerging dimensions of analysis

\begin{tabular}{|c|c|c|c|c|c|}
\hline Network Type & $\begin{array}{l}\text { MNC (US-Global) } \\
\text { - Product Assembler }\end{array}$ & $\begin{array}{l}\text { MNC (US-Regional) } \\
\text { - Product Assembler }\end{array}$ & $\begin{array}{l}\text { MNC (US-EU) } \\
\text { - Product Assembler }\end{array}$ & $\begin{array}{l}\text { SME (UK) - Product } \\
\text { Assembler }\end{array}$ & $\begin{array}{l}\text { SME (UK) - Component } \\
\text { Manufacturer }\end{array}$ \\
\hline Network Phase & Mature & Emerging & Nascent & Emerging & Nascent \\
\hline Network Stage & Established & Expansion $->$ Stabilisation & Formation -> Expansion & Fragmented -> Formation & Embryonic \\
\hline $\begin{array}{l}\text { Product } \\
\text { Form } \\
\text { Maturity } \\
\text { Configuration }\end{array}$ & $\begin{array}{l}\text { Mature product line; No } \\
\text { product design difference } \\
\text { between location and 'lead' } \\
\text { location product portfolios }\end{array}$ & $\begin{array}{l}\text { Emerging/established } \\
\text { product line; Modular } \\
\text { product design changes } \\
\text { between location and 'lead' } \\
\text { location product portfolios }\end{array}$ & $\begin{array}{l}\text { New/emerging product } \\
\text { line; Different product } \\
\text { designs between location } \\
\text { and 'lead' location product } \\
\text { portfolios }\end{array}$ & $\begin{array}{l}\text { Alpha product lock-in; } \\
\text { Proof-of-concept }\end{array}$ & $\begin{array}{l}\text { Consolidation of concepts, } \\
\text { technical flexibility; Licencing }\end{array}$ \\
\hline $\begin{array}{l}\text { Structure } \\
\text { Organisational Context } \\
\text { Capabilities (Technology } \\
\text { transfer experience; } \\
\text { Levels of knowledge and } \\
\text { expertise; absorptive } \\
\text { capacity) }\end{array}$ & $\begin{array}{l}\text { Fully established Knowledge } \\
\text { Network Globally; } \\
\text { Technology transfer } \\
\text { experience (including } \\
\text { previous transfer of } \\
\text { production line to other } \\
\text { locations); Sufficient } \\
\text { absorptive capacity } \\
\text { (demonstrated } \\
\text { independently) }\end{array}$ & $\begin{array}{l}\text { Established Knowledge } \\
\text { Network between 'lead } \\
\text { location' and case study } \\
\text { location; Evidence of } \\
\text { previous technology } \\
\text { transfers (other product } \\
\text { lines); Often sufficient } \\
\text { absorptive capacity } \\
\text { (demonstrated only when } \\
\text { requested) }\end{array}$ & $\begin{array}{l}\text { Limited Knowledge } \\
\text { Network between 'lead } \\
\text { location' and case study } \\
\text { location; } 1^{\text {st }} \text { technology } \\
\text { transfer in progress; } \\
\text { Limited levels of } \\
\text { knowledge and expertise; } \\
\text { Insufficient absorptive } \\
\text { capacity }\end{array}$ & $\begin{array}{l}\text { Actors for development and } \\
\text { production } \\
\text { Increasing visibility on } \\
\text { potential partners who cover } \\
\text { the entire value chain and } \\
\text { neighbouring sectors. } \\
\text { Leveraging innovation centres } \\
\text { responsible for building } \\
\text { regional open-innovation } \\
\text { communities }\end{array}$ & $\begin{array}{l}\text { Initial partnership } \\
\text { arrangement with potential } \\
\text { customer/suppliers } \\
\text { Growing an awareness of } \\
\text { e.g. innovation centres, who } \\
\text { have established networks }\end{array}$ \\
\hline $\begin{array}{l}\text { Network Dynamics } \\
\text { Production Line }\end{array}$ & Fully automated & Semi-automated & $\begin{array}{l}\text { Labour intensive, limited } \\
\text { automation }\end{array}$ & Batch to Continuous Flow & Continuous flow \\
\hline $\begin{array}{l}\text { Governance and } \\
\text { Coordination } \\
\text { Production Control (KPIs) } \\
\text { External influences }\end{array}$ & $\begin{array}{l}\text { Unified and Global KPIs, } \\
\text { international comparisons; } \\
\text { Limited external influence } \\
\text { (apart from e.g. safety } \\
\text { regulations) }\end{array}$ & $\begin{array}{l}\text { Comparison of production } \\
\text { KPIs (location v. 'lead' } \\
\text { location); Moderate level of } \\
\text { external influence (labour } \\
\text { incentives) }\end{array}$ & $\begin{array}{l}\text { Isolated and individual } \\
\text { KPIs in production plant; } \\
\text { High level of external } \\
\text { (government) influence (IP } \\
\text { incentives) }\end{array}$ & $\begin{array}{l}\text { Determining what success } \\
\text { look like (measurement) }\end{array}$ & $\begin{array}{l}\text { Focus on managing internal } \\
\text { capabilities }\end{array}$ \\
\hline $\begin{array}{l}\text { Support Infrastructure } \\
\text { Language (Organisational, } \\
\text { native tongue) }\end{array}$ & $\begin{array}{l}\text { Fully established } \\
\text { organisational language } \\
\text { adopted globally }\end{array}$ & $\begin{array}{l}\text { Consistent documentation, } \\
\text { some language and } \\
\text { communication barriers }\end{array}$ & $\begin{array}{l}\text { Leveraging knowledge } \\
\text { cross-category/region. } \\
\text { People will be starting } \\
\text { from different points }\end{array}$ & $\begin{array}{l}\text { Recognising different cultures } \\
\text { Putting support Infrastructure } \\
\text { in place }\end{array}$ & $\begin{array}{l}\text { Developing a common } \\
\text { understanding/language v. } \\
\text { different interpretations }\end{array}$ \\
\hline $\begin{array}{l}\text { Relationships } \\
\text { Intensity of connection } \\
\text { Contact frequency } \\
\text { Power distance } \\
\text { Masculinity (individualism) }\end{array}$ & $\begin{array}{l}\text { Close relationships (defined } \\
\text { roles and responsibilities); } \\
\text { Established and regular } \\
\text { routine of meetings } \\
\text { scheduled (full participation); } \\
\text { Perception of symmetrical } \\
\text { relationships (equal } \\
\text { partners); Close cooperation } \\
\text { (global interest) }\end{array}$ & $\begin{array}{l}\text { Growing relationships } \\
\text { (partial understanding of } \\
\text { who is who and who } \\
\text { does/knows what); Irregular } \\
\text { meetings (limited availability } \\
\text { and participation); } \\
\text { perception of un-even } \\
\text { relationship v. 'lead' location; } \\
\text { "Healthy" competition (mix of } \\
\text { location and global interests) }\end{array}$ & $\begin{array}{l}\text { Selecting the correct } \\
\text { people who can deliver on } \\
\text { the skills and capabilities } \\
\text { albeit with effective } \\
\text { training }\end{array}$ & $\begin{array}{l}\text { Assessing Innovation and } \\
\text { education activities that have a } \\
\text { structured mentoring and } \\
\text { networking programme }\end{array}$ & Focus on delivery \\
\hline
\end{tabular}


Table 8. Knowledge Mobility Configuration Profile - Knowledge Transfer Mechanisms

\begin{tabular}{|c|c|c|c|c|c|}
\hline Network Type & $\begin{array}{l}\text { MNC (US-Global) } \\
\text { - Product Assembler }\end{array}$ & $\begin{array}{l}\text { MNC (US-Regional) } \\
\text { - Product Assembler }\end{array}$ & $\begin{array}{l}\text { MNC (US-EU) } \\
\text { - Product Assembler }\end{array}$ & $\begin{array}{l}\text { SME (UK) - Product } \\
\text { Assembler }\end{array}$ & $\begin{array}{l}\text { SME (UK) - Component } \\
\text { Manufacturer }\end{array}$ \\
\hline Network Phase & Mature & Emerging & Nascent & Emerging & Nascent \\
\hline Network Stage & Established & Expansion $->$ Stabilisation & Formation -> Expansion & Fragmented $->$ Formation & Embryonic \\
\hline $\begin{array}{l}\text { Transfer of Fundamental } \\
\text { Knowledge }\end{array}$ & $\begin{array}{ll}\text { - } & \text { Global Boundary } \\
\text { - } & \text { Spanner } \\
\text { Global audit of } \\
\text { production plants } \\
\text { (partial) } \\
\text { - } \\
\text { Global and } \\
\text { established standard } \\
\text { operating procedures } \\
\text { (SOPs) } \\
\text { - Electronic linkages } \\
\text { for Global } \\
\text { communication } \\
\end{array}$ & $\begin{array}{ll}\text { - } \quad \text { 'Lead Location' } \\
\text { Boundary Spanner } \\
\text { - Global audit of } \\
\text { production plants } \\
\text {-Lead location' } \\
\text { standard operating } \\
\text { procedures (SOPs) } \\
\text { Electronic linkages } \\
\text { for Global } \\
\text { communication }\end{array}$ & $\begin{array}{ll}\text { - } & \text { 'Lead Location' } \\
\text { Boundary Spanner } \\
\text { (partial) } \\
\text { - } \quad \text { Local audit } \\
\text { - } \quad \text { Hybrid of individual } \\
\text { and copied standard } \\
\text { operating procedures } \\
\text { (SOPs) (partial) } \\
\text { - Electronic linkages } \\
\text { for communication } \\
\text { with 'lead' location }\end{array}$ & $\begin{array}{l}\text { Electronic linkages } \\
\text { for communication }\end{array}$ & $\begin{array}{l}\text { Many issues of } \\
\text { knowledge integration } \\
\text { may be dealt with } \\
\text { relatively simply, and } \\
\text { even informally }\end{array}$ \\
\hline $\begin{array}{l}\text { Transfer of Moderate } \\
\text { Knowledge }\end{array}$ & $\begin{array}{ll}\text { - } & \text { International forum - } \\
\text { face-to-face meetings } \\
\text { of Global experts } \\
\text { (partial) } \\
\text { International teams } \\
\text { (global team located } \\
\text { at 'lead location') } \\
\text { Global and } \\
\text { established best } \\
\text { practice guidelines } \\
\text { - Global benchmarking } \\
\text { of all production } \\
\text { plants (partial) }\end{array}$ & $\begin{array}{ll}\text { - } & \text { International forum - } \\
\text { face-to-face meetings } \\
\text { of Global experts } \\
\text { (partial) } \\
\text { - International teams } \\
\text { (global team located } \\
\text { at 'lead location') } \\
\text { (partial) } \\
\text { 'Lead location' } \\
\text { established best } \\
\text { practice guidelines } \\
\text { (partial) } \\
\text { Access to benchmark } \\
\text { reports }\end{array}$ & $\begin{array}{ll}\text { - } & \text { Meeting of production } \\
\text { plant and 'lead' } \\
\text { location experts } \\
\text { (partial) } \\
\text { - International teams } \\
\text { (global team located } \\
\text { at 'lead location') } \\
\text { 'Lead location' } \\
\text { established best } \\
\text { practice guidelines } \\
\text { (partial) } \\
\text { - Access to benchmark } \\
\text { reports (partial) }\end{array}$ & $\begin{array}{l}\text { - Leveraging MNC } \\
\text { network to gain } \\
\text { professional training } \\
\text { combined with } \\
\text { hands-on experience } \\
\text { Emerging assets } \\
\text { increasingly utilised } \\
\text { in this space e.g. } \\
\text { SPOCs (Specialized } \\
\text { Private Online } \\
\text { Courses) }\end{array}$ & $\begin{array}{l}\text { - Affordable access to } \\
\text { targeted and meaningful } \\
\text { education and training in } \\
\text { particular domain } \\
\text { Massive Open Online } \\
\text { Courses (MOOCs) }\end{array}$ \\
\hline $\begin{array}{l}\text { Transfer of Advanced } \\
\text { Knowledge }\end{array}$ & $\begin{array}{ll}\text { - } & \text { Expatriation of Global } \\
\text { experts at production } \\
\text { location (partial) } \\
\text { Overseas Training at } \\
\text { 'lead location' } \\
\text { (partial) }\end{array}$ & $\begin{array}{ll} & \text { Expatriation of 'lead } \\
\text { plant' experts at } \\
\text { production location } \\
\text { Overseas Training at } \\
\text { other Global } \\
\text { production locations }\end{array}$ & $\begin{array}{ll}- & \text { Expatriation of 'lead } \\
\text { plant' experts at } \\
\text { production location } \\
\text { Overseas Training at } \\
\text { 'lead location' }\end{array}$ & $\begin{array}{ll}\text { Interactive SPOCs } \\
\text { tailored to the specific } \\
\text { demands of the SME } \\
\text { Rotating Elevator } \\
\text { Workshops with } \\
\text { network partners }\end{array}$ & $\begin{array}{l}\text { - 'Facilitator' role often } \\
\text { useful } \\
\text { Moodle as a flexible } \\
\text { Virtual Learning and } \\
\text { knowledge exchange tool }\end{array}$ \\
\hline
\end{tabular}


Our findings demonstrate the critical role of knowledge management in internationalisation, and that skills, knowledge, technology and organisational processes are integral to any emerging network design criteria and/or 'capability' acquisition assessment.

The framework extends the network configuration approach to incorporate knowledge-specific dimensions of analysis and associated knowledge integration mechanisms, derived from the academic literature and practice. The literature review identified key gaps and themes, which included: the need for a common framework that captures the overall structure of knowledge transfer frameworks, providing a holistic view of all dimensions and their relationship (existing research tends to focus on a specific aspect of the knowledge mobility process); a better understanding of the different aspects of knowledge, and different dimensions of the network configuration and their relationship, overview of best practices mechanisms, and what knowledge types they transfer most effectively. The choice of integration mechanism is not only influenced by the type of knowledge being 'mobilised', but also by industrial context and the maturity of the network within which the knowledge is being shared.

This nuanced approach has been used successfully as a basis for a proactive and differentiated approach to knowledge mobility within any network. In summary, between the recipient and source, the knowledge context element of the refined framework requires:

- Knowledge Level: Level of knowledge to be transferred

- Knowledge Characteristics: Dimensions defining the type of knowledge

- Knowledge Package: the message which has to be delivered

- Internalised Knowledge Package: Level of knowledge recipient has acquired

Between the recipient and source, the network context element of the framework consists of conventional dimensions, plus emerging dimensions, from the knowledge literature, in terms of: Structure; Network Dynamics; Relationships; Governance and Coordination; Maturity Level.

Integrating these contexts, figure 8 sets out the final Knowledge Mobility Configuration framework, which captures the critical role of knowledge context, network configuration and transfer mechanism in the success of the transfer. Here, between the recipient and source, the knowledge transfer mechanisms best employed to support different network configurations should be used to transfer different types of knowledge. 


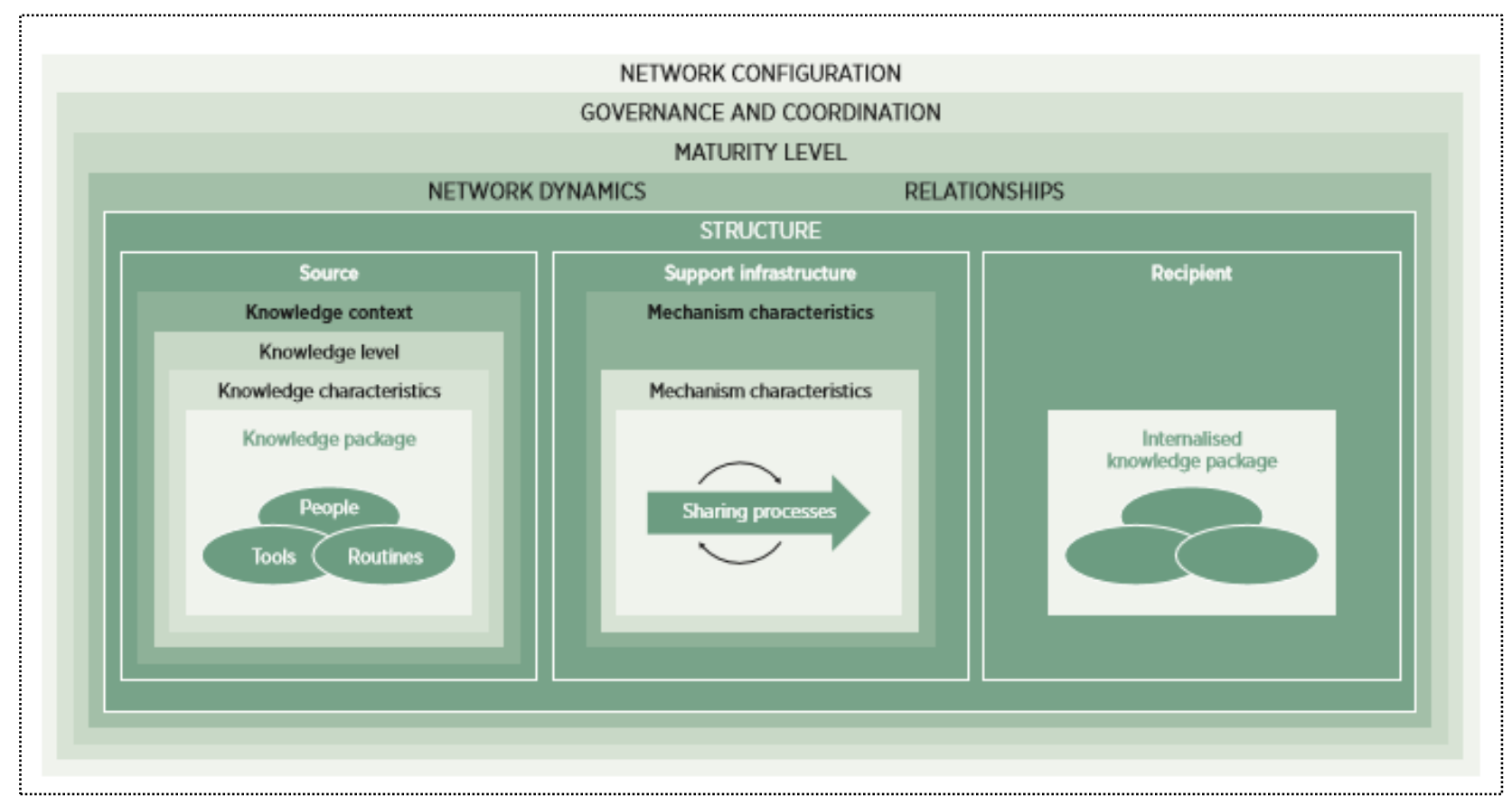

Figure 8. Knowledge Mobility Configuration framework: Network configuration framework extended to incorporate knowledge-specific dimensions of analysis and associated knowledge integration mechanisms, derived from the academic literature and practice

The refined framework serves to reduces complexity, and combines both configuration aspects of the knowledge network, as well as insights on optimum knowledge transfer mechanisms in practice today - offering a visual representation of the current state of the knowledge transfer network configuration within an organisation or network. Our results suggest that there is an active knowledge-sharing network and that multiple levels of employees understand the requirement for such a network and how their line integrates with the network. In summary:

- Knowledge configuration profiles capture the knowledge context, describing the knowledge level and their characteristics, the network configuration in terms of product maturity and their appropriate knowledge mobility mechanism.

- Maturity of the product greatly influences the success of transfers, and the more established the network the more streamlined the knowledge transfer process.

- Structure, abilities and relationship status of the transfer parties greatly impact the knowledge sharing process.

- Different knowledge mobility mechanisms will be better suited then others for the transfer of different knowledge characteristics and levels.

- Depending on the network configuration, different knowledge sharing mechanisms will be available and can be designed to fit specific purposes (for example, concepts of operation for 'virtual teams' versus communities of practice). 
As every company has its own culture, dimensions may need to be defined using an internally accepted company language. There are limitations to the user's level of influence, as some stakeholders will be able to influence different dimensions more than others that need to be better understood. Our research supports the assertion that absorptive capacity, the ability and capability of organisations to make use of external knowledge is an important phase of the process and that the effectiveness of absorption, embedding and diffusion may have an influence in selecting and managing future collaborations. It was found that SMEs often have limited resources in knowledge management and need confidence to adopt any initiative, which can be built up by improved approaches tested in SMEs. Knowledge transfer here may be enhanced through the adoption of collaborative resource sharing, and cross-pollination of knowledge, even when the supplier is 'locked-in' to a customer and not co-supplying a competitor.

\section{Conclusions and directions for future research}

Knowledge management theories and practices that are implemented in large organisations may not always be best suited to smaller companies. This study addresses a need to conduct empirical research to develop theoretical frameworks for a deeper understanding and a platform for the future development of the field. The methodology, developed as part of this study, focuses on the capture of tacit knowledge and the establishment of dynamic knowledge transfer network configurations.

By examining knowledge mobility mechanisms within the context of network maturity, the research examines how both the knowledge task and also the available knowledge mobility mechanism are modified by network maturity and industry context. A literature review within the domains of knowledge transfer and network configuration revealed a research gap in the exploration of knowledge 'mobility', in terms of network configuration. Existing literature remains largely descriptive and only considers a very narrow window when looking at the different dimensions affecting the transfer. In order to address this research gap this study looked to explore how best to capture knowledge configuration profiles in MNCs and SMEs.

The extant literature revealed different approaches discussing the dimensions of knowledge sharing and how they promoted or inhibited transfer effectiveness. The approach of Cumming (2003) formed the basis for a prototype framework, which used emerging dimensions specific to knowledge and transfer characteristics, as well as stages of network emergence. The knowledge mobility configuration profile of five case studies captured current (and future desired) states of knowledge management within the networks under investigation

This research has made valuable contributions to the analysis of knowledge: analysing the different determinants of knowledge networks and demonstrating how they support or enable sharing processes. This research unites the different determinants of knowledge mobility, providing a holistic understanding of the dimensions, their relationship and implementation within different network configurations and maturity levels. In summary: 
- The overview of the different dimensions and sub-dimensions of knowledge network processes enables a better understanding of their different characteristics, and their relationship affecting knowledge transfer activities.

- The network configuration element of the KMC framework provides insights on how global product networks mature, and how their knowledge mobility profiles may evolve over time.

- The characterisation of different mechanisms for each stage of emergence informs existing literature and provides an understanding not only on which mechanism are applied within industry also how these mechanisms are utilised within different network maturity levels.

One of the benefits of applying research in practice is that it helps identify and solve problems (both new and existing) that originate from industry (Childe, 2011). Here, application of the $\mathrm{KMC}$ framework contributes to practice in several ways, for example:

- A very complex matter can be absorbed in a very short time.

- A visual picture of the current state configuration (capturing the results) that everyone can access (codification).

- Mapping different perspectives, against a future desired state, provides an overview where they are aligned or not, highlighting areas of concern.

- Provides a basis for benchmark activities, as the current state can be mapped against a future current state, where improvements as well as drawbacks can be outlined. For example, key insights across the cases centred on: what are the key activities to be performed, processes key to integration, 'linkages' between locations; alignment on priorities and goals; and empowerment.

There are a series of limitations to this study, which present interesting opportunities for future research. In specifically examining knowledge transfer mechanisms in knowledge-intensive manufacturing firms (and across their networks), first, is the nature of the case studies and access to a significant number of case examples. While the KMC framework was first developed using an extensive literature review, validation and insights were restricted to five case studies (with supporting secondary data) across a knowledge-critical sector. Access was limited to two SMEs and three OEMs at the time of framework application. This is understandable given sensitivities over IP and high rates of turnover/attrition, with respect to respondents and smaller firms. However, the selection criteria set out did allow a diversity of enterprises operating at different (and multiple) points across the value chain to be identified and targeted. In summary, additional validation with a more extensive set of cases would be beneficial. A second limitation is about the research design, particularly the unit of analysis. Three of the cases are networks of the same organisation, and this research treated them as individual cases, because they are fairly independent at both strategic and operational levels. With knowledge being obtained through organisational task and in specific settings, it may be 
unique to individual organisations. Hence, these three case networks may be strongly influenced by the culture and orientations of the organisation's central function. However, as this research involved interviewing managers and engineers from different parts of the business this was beneficial in obtaining a comprehensive view of knowledge management for the organisation and its network operations.

Despite these limitations, engagement with industry served to identify the practice need for new avenues of research. Directions for future research are suggested here based on the above discussions.

One area of focus is to capture generic configuration patterns or archetypes of Knowledge Mobility through more studies in a broader range of industry sectors, and to enable refinement of the dimensions of analysis.

As part of an emerging technology research agenda, we will also examine how ITenabled and e-commerce-based supply chains are changing the roles of information and knowledge, in addition to the future role of Knowledge Information Centres (KICs). SMEs are regarded as the 'backbone' in many sectors and, as a result, KICs are developing strategies to include a significant number of SMEs in various activities. KICs, themselves, could be regarded as start-ups with the complexity of a MNC, and a future focus of research will explore knowledge mobility mechanisms in regional open-innovation communities that support, catalyse and accelerate the embedding of innovation in SMEs, and across MNCSME networks.

\section{REFERENCES}

Aboelmaged, M.G. 2014. "Linking operations performance to knowledge management capability: the mediating role of innovation performance." Production Planning and Control 25 (1): 44-58.

Alavi, M., and D.E. Leidner. 2001. "Review: KM and KM systems: conceptual foundations and research issues." MIS Quarterly, 25(1): 107-136.

Alexander, A.T., and S.J. Childe. 2013. "Innovation: a knowledge transfer perspective." Production Planning and Control 24 (2-3): 208-225.

Allen, T. J. 1977. "Managing the flow of technology: Technology transfer and the dissemination of technological information within the R\&D organization" Cambridge, MA: MIT Press.

Almeida, P., and R. Grant, R. 1998. "International Corporations and Cross-border KT in the Semiconductor Industry" Carnegie Bosch Institute for Applied Studies in International Management Working Paper 98-13, Graduate School of Industrial Administration, Carnegie Mellon University

Almeida, P., and B. Kogut. 1999. "Localization of knowledge and the mobility of engineers in regional networks." Management Science 45: 905-917.

Almeida, P., and A. Phene. 2004. "Subsidiaries and knowledge creation: the influence of the MNC and host country on innovation." Strategic Management Journal 25: 847-864. 
Ambrose E., D. Marshall, and D. Lynch. 2010. "Buyer supplier perspectives on supply chain relationships." International Journal of Operations and Production Management 30 (12): 1269-1290.

Andrews, K. 1971. The concept of strategy, Homewood, IL: Irwin.

Appleyard .1996). "How does knowledge flow? Interfirm patterns in the semiconductor industry." Strategic Management Journal 17: 137-154.

Argote, L. 1999. "Organizational learning: Creating, retaining and transferring knowledge". Norwell, MA: Kluwer.

Argyris, C. 1990. "Overcoming organizational defenses: Facilitating organizational learning." Boston: Allyn and Bacon.

Ates, A., P. Garengo, P. Cocca, and U. Bititci. 2013. "The development of SME managerial practice for effective performance management." Journal of Small Business and Enterprise Development 20(1): 28-54.

Babbie, E. 1989. "The practice of Social Research." 5th ed. Belmont, Wadsworth.

Baliga, B. and A. Jaeger. 1984. "Multinational corporation: Control systems and delegation issues." Journal of International Business Studies 25-40.

Bandura, A. 1986. "Social foundations of thought and action." Englewood Cliffs, NJ: Prentice Hall.

Barney, J. 1991. "Organization resources and sustained competitive advantage" Journal of Management 17: 99-120.

Berryman, Reba. (2005). KM in virtual organizations: A study of a best practices KT model. Doctor of Philosophy (Applied Technology, Training and Development)

Birkinshaw, J. 2001. "Why is KM so difficult?" Business Strategy Review 12(1): 11-18.

Bresman, H., et al. 1999. "KT in international acquisitions." Journal of International Business Studies 30 (3): 439-462.

Cerchione R., E. Esposito, and M.R. Spadaro. 2015. "The spread of knowledge management in SMEs: A scenario in evolution" Sustainability 7: 10210-10232.

Chai, K., et al. 2003. "Bridging islands of knowledge: a framework of KS mechanisms"; International Journal of Technology Management 25(8): 703- 727.

Chen, J., and R. McQueen. 2008. "KT processes for different experience levels of knowledge recipients at an offshore technical support center." Information Technology and People 23 (1): 54-79.

Chiesa, V., and R. Manzini. 1996. "Managing KT within multinational firms." International Journal of Technology Management 12 (4): 462-475.

Childe, S.J. 2011 "Case studies in operations management" Production Planning and Control 22 (2): 107.

Cohen, W., and D. Levinthal. 1990. "Absorptive capacity: A new perspective on learning and innovation” Administrative Science Quarterly 35(1): 128-152. 
Cook, S., and J. Brown. 1999. "Bridging epistemologies: The generative dance between organizational knowledge and organizational knowing." Organization Science 10 (4): 381-400.

Cowan, R., and D. Foray. 1997. "The economics of codification and the diffusion of knowledge" Industrial and Corporate Change 6: 595-622.

Cullen, J.B. 2002. "Multinational Management: A Strategic Approach." South-Western Publishers, Cincinnati, $\mathrm{OH}$.

Cummings, J. 2003. "KS: A Review of the Literature." The World Bank Operations Evaluation Department. Washington, D.C.

Davenport, E. 2002." Mundane KM and microlevel organizational learning: An ethological approach." Journal of the American Society for Information Science and Technology 53 (12): 1038-1046.

Davenport, T., and L. Prusak. 1998. "Working knowledge: How organizations manage what they know." Boston: Harvard Business School Press.

Davenport, T. and L. Prusak. 2000. "Working Knowledge - How Organizations Manage what They Know." Harvard Business School Press, Boston, MA.

Davenport T, et al. 1996. "Improving knowledge work processes." Sloan Management Review 37: 53-65.

D'Este, P., and P. Patel. 2007. "University-industry linkages in the UK: What are the factors underlying the variety of interactions with industry?" Research Policy, 36(9), 12951313.

Deitz, A., and S. Ellershaw. 1999 "I'm not in the knowledge business ... am I?" In M. Bullock, C. Friday, K. Belcher, and B. Bisset (Eds.), International Conference on Work Teams Proceedings 237-241. Denton: University of North Texas.

Delbufalo, E. 2017. 'The effects of suppliers' trust on manufacturers' innovation capability: an analysis of direct versus indirect relationships." Production Planning and Control 28 (14):

De Meyer, A. 1991 "Tech Talk: how managers are stimulating global R\&D communication." Sloan Management Review 32 (3): 49-58.

Di Gregorio, D., M. Musteen and D.E. Thomas. 2012 "Offshore outsourcing as a source of international competitiveness for SMEs." Journal of International Business Studies 40, 969-988.

Dixon, N. 1994. "The Organizational Learning Cycle: How We Can Learn Collectively." New York: McGraw-Hill

Dixon, N. 2000. "Common knowledge: How companies thrive by sharing what they know." Boston: Harvard Business School Press.

Dooley, L., D. Kirk, and K. Philpott. 2013. "Nurturing life-science knowledge discovery: managing multi-organisation networks" Production Planning and Control 24 (2-3): 195-207

Doz, Y. 1996. "The evolution of cooperation in strategic alliances: Initial conditions or learning processes?" Strategic Management Journal 17: 55-83. 
Doz, Y. and C. Prahalad, C. 1991. "Managing DMNCs: A search for a new paradigm." Strategic Management Journal, 12: 145-164.

Doz, Y. and J. Santos, J. 1997 'On the management of knowledge: from the transparency of collocation and co-setting to the quandary of dispersion and differentiation," Insead Working Paper No. 97/119/SM. INSEAD, France.

Dreyfus, H. and S. Dreyfus, S. 1986. "Why computers may never think like people." Technology Review 89 January.

Drucker, P. 1999. "Management challenges for the 21st century. "New York: HarperCollins.

Durst, S., and I. Runar Edvardsson. 2012 "Knowledge management in SMEs: a literature review." Journal of Knowledge Management, 16 (6): 879-903.

El Sawy, O. et al. 1998. "Understanding the nature of shared knowledge creation spaces around business processes: an international investigation." Working paper, University of Southern California, Los Angeles, CA.

Ellram, L.M., and A. Carr. 1994. "Purchasing: a history and review of the literature." International Journal of Purchasing and Materials Management 30 (2): 10-18.

Enright, M. 2000. "Regional clusters and multinational enterprises." International Studies of Management and Organization 30: 114-138.

Etzioni, A. 1961. "A comparative analysis of complex organizations." New York: Free Press.

Fosfuri, A. and J. Tribo. 2008. "Exploring the antecedents of potential absorptive capacity and its impact on innovation performance." Omega, 36(2): 173-187.

Fleet, D., T.S. Harrington, and J.S, Srai. 2014. "Knowledge Integration”, in, 'Capturing value from global networks: strategic approaches to designing international production, supply and service operations', University of Cambridge, Institute for Manufacturing. ISBN: 978-1-902546-30-8.

Galbraith, C. 1990. "Transferring core manufacturing technologies in high technology firms." California Management Review, 32 (4): 56-70.

Gargiulo, M., and M. Benassi. 2000. "Trapped in your own net: network cohesion, structural holes, and the adaptation of social capital," Organization Science 11: 183-201.

Garud, R., and P. Nayyar. 1994. "Transformative capacity: Continual structuring by intertemporal technology transfer." Strategic Management Journal 15: 365-385.

Gold, A. et al. 2001. "KM: An organizational capabilities perspective." Journal of Management Information Systems 18(1): 185-214.

Goold, M., and A. Campbell. 2003. "Making Matrix Structures Work: Creating Clarity on Unit Roles and Responsibility." European Management Journal (21) 3: 351-363.

Graham, J. 1985. "The influences of culture on business negotiations," Journal of International Business Studies 16: 81-96.

Granovetter, M. 1985. "Economic action and social structure: the problem of embeddedness." American Journal of Sociology 91: 481-510.

Grant, R. 1996. "Toward a knowledge-based theory of the firm." Strategic Management Journal 17: 109-122. 
Grant, E. 1999. "Fitness for transfer - assessing manufacturing technologies for relocation." University of Cambridge, Institute for Manufacturing. ISBN 1-902546-01-6

Guinery, J., and B. MacCarthy. 2009. "Managing key interfaces in production planning and control." Production Planning and Control 20 (1): 40-56

Gupta, A., and V. Govindarajan. 2000. "Knowledge flows within multinational corporations." Strategic Management Journal 21: 473-496.

Gunasekaran, A., L. Forker, and B. Kobu. 2000. "Improving operations performance in a small company: a case study." International Journal of Operations and Production Management 20 (3): 316-336.

Lee, S. and R.D. Klassen. 2008. "Drivers and Enablers That Foster Environmental Management Capabilities in Small - and Medium-Sized Suppliers in Supply Chains." Production and Operations Management, 17 (6): 573 - 586.

Minshall, T. 1999. "Manufacturing mobility - a strategic guide to transferring manufacturing capability." University of Cambridge, Institute for Manufacturing. ISBN 978-1-90254602-5

Pongpanich, C. 2000. "Manufacturing location decisions - choosing the right location for international manufacturing facilities." University of Cambridge, Institute for Manufacturing. ISBN 978-1-902546-05-6

Hamel, G. 1991. "Competition for competence and inter-partner learning within international strategic alliances." Strategic Management Journal 12: 83-103.

Hansen, M. 1999. "The search-transfer problem: The role of weak ties in sharing knowledge across organization subunits." Administrative Science Quarterly

Hansen, M.T., and J. Birkinshaw. 2007. "The innovation value chain." Harvard Business Review 85 (6): 121-130.

Harrington, T.S., D.A, Kirkwood, and J.S. Srai. 2012. "Performance metric selection methodology for multi-organizational service network integration," Journal of Applied Management and Entrepreneurship 17 (3): 20-35.

Harrington, T.S., J.S. Srai, and P. Christodoulou. 2015. "Connecting the unconnected: new thinking on next-generation supply chains." IfM Review 4: 24-26.

Harrington, T.S., and J.S. Srai. 2016. "Designing a 'concept of operations' architecture for next-generation multi-organisational service networks." Al and Society: Journal of Knowledge, Culture and Communication. Published online: 17 May. DOI: 10.1007/s00146-016-0664-5

Harrington, T.S., J.S. Srai, M. Kumar, and J. Wohlrab. 2016. "Identifying design criteria for urban system 'last-mile' solutions - a multi-stakeholder perspective." Production Planning and Control 27 (6): 456-476.

Harrington, T.S., and J.S. Srai. 2017. "Understanding stages of supply network emergence in technology commercialisation." International Journal of Manufacturing Technology and Management 31 (1-3): 4-36. 
Harrington, T.S., M.A. Phillips, and J.S. Srai. 2017. "Reconfiguring global pharmaceutical value networks through targeted technology interventions." International Journal of Production Research 55 (5): 1471-1487.

Hartley, J. 2004. "Case study research", In Catherine Cassell \& Gillian Symon (Eds), Essential guide to qualitative methods in organizational research. London: Sage.

Hedberg, B. 1981. "How organizations learn and unlearn," in P.C. Nystrom and W. H. Starbucks, editors, Handbook of organizational design, Oxford: Oxford University Press.

Hofstede, G. 1980. "Culture's consequences: International differences in work-related values." Beverly Hills, CA: Sage Publications.

Hofstede, G. 1997. "Cultures and Organizations: Software of the Mind." revised edition, McGraw-Hill, New York, NY.

Hofstede, G. 2001. "Culture's Consequences." Sage, Beverly Hills, CA.

lansiti, M. 1998. "Technology integration: making critical choices in a dynamic world." Boston: Harvard Business School Press.

Jacobs, J. 1969. "The economy of cities." New York: Random House.

Kedia, B., and R. Bhagat. 1988. 'Cultural constraints on transfer of technology across nations: implications for research in international and comparative management." Academy of Management Review 13 (4): 559-571.

Kim, L., and R. Nelson. 2000. "Technology, learning, and innovation: Experiences of newly industrializing economies." Cambridge, UK: Cambridge University Press.

Kostova, T. 1996. "Success of the transnational transfer of organizational practices within multinational corporations", unpublished doctoral research, University of Minnesota, Minneapolis, MN.

Kostova, T. 1999. "Transnational transfer of strategic organizational practices: A contextual perspective." Academy of Management Review 24 (2): 308-324.

Kuprenas, J.A. 2003. "Implementation and performance of a matrix organisation structure." International Journal of Project Management 21(1): 51-62.

Lam, A. 1997. "Embedded firms, embedded knowledge: problems of collaboration and KT in global cooperative ventures." Organization Studies 18 (6): 973-996.

Lam, A. 2000. "Tacit knowledge, organizational learning and societal institutions: an integrated framework." Organization Science 21 (3): 487-513.

Landryová, L., and C. Irgens. 2006. "Process knowledge generation and knowledge management to support product quality in the process industry by supervisory control and data acquisition (SCADA) open systems" Production Planning and Control 17 (2): 94-98

Lee, A. 1991. "Integrating Positivist and Interpretive Approaches to Organizational Research." Organization Science 2(4): 342-365.

Lehr, J., and R. Rice. 2002. "Organizational measures as a form of KM: A multitheoretic, communication-based exploration." Journal of the American Society for Information Science and Technology 53(12): 1060-1073. 
Lev, B. 2001. "Intangibles: Management, Measurement, and Reporting." Brookings Institute: Washington, DC.

Liu, S., J. Moizer, P. Megicks, D. Kasturiratne, and U. Jayawickrama. 2014. "A knowledge chain management framework to support integrated decisions in global supply chains" Production Planning and Control 25 (8): 639-649

Lucas, L., 2006 "The role of culture on KT: the case of the multinational corporation." The Learning Organization 13 (3): 257-275

Nagati, H., and C. Rebolledo. 2013 "Improving operational performance through knowledge exchange with customers" Production Planning and Control 24 (8-9): 658-670

Nonaka, I. 1994. "A dynamic theory of organizational knowledge creation." Organization Science 5 (1): 14-36.

Nonaka, I., and H. Takeuchi. 1995. "The Knowledge-Creating Company." Oxford University Press, New York.

Novins, P., and R. Armstrong. 1998. "Choosing your spots for KM" Perspectives on Business Innovation 1: 45-54.

O'Dell, C., and C. Grayson. 1998. "If only we knew what we know: The transfer of internal knowledge and best practice." New York: The Free Press.

Ouchi, W. 1980. "Markets, bureaucracies, and clans." Administrative Science Quarterly 25: 129-141.

Pawar, K.S., and H. Rogers. 2014. "Mobilising knowledge across organisational boundaries: addressing human issues in the telecommunications industry." Production Planning and Control 25 (5): 355-371

Peteraf, M., and M. Shanley. 1997. "Getting to know you: A theory of strategic group identity." Strategic Management Journal, 18: 165-186.

Pfeffer, J., and R. Sutton. 2000. "The knowing-doing gap: How smart companies turn knowledge into action" Boston: Harvard Business School Press.

Phene, A. et al. 2005. "KT within the multinational firm: what drives the speed of transfer?" Management International Review 45: 53-74.

Phillips, M.A., T.S. Harrington, and J.S. Srai. 2017. "Convergent innovation in emerging healthcare technology ecosystems: addressing complexity and integration", Technology Innovation Management Review (in print)

Pisano, G. 1994. "Knowledge integration and the locus of learning: An empirical analysis of process development." Strategic Management Journal 15: 85-100.

Polanyi, M. 1966. "The Tacit Dimension.” Routledge \& Kegan Paul, London.

Powell, W. et al. 1996. "Interorganizational collaboration and the locus of innovation: Networks of learning in biotechnology." Administrative Science Quarterly 41: 116-145.

Premkumar, G.P. 2006. "Interorganizational systems and supply chain management: An information processing perspective." Information and Management, 43 (3):395-408.

Prusak, L. 1999. "Action review of KM: Report and recommendations." World Bank. 
Remenyi, D. 1995. "So you want to be an academic researcher in business and Management studies! Where do you start and what are the key philosophical issues to think about?" Working Paper Series

Rogers, E.M. 2003. "Diffusion of Innovations." 5th ed., The Free Press, New York.

Romero, D., and A. Molina. 2011. "Collaborative networked organisations and customer communities: value co-creation and co-innovation in the networking era." Production Planning and Control 22 (5-6): 447-472.

Ruggles, R. 1998. "The state of the notion: KM in practice." California Management Practice 40(3): 80-89.

Sagafi-nejad, T. 1990. "International technology transfer literature: Advances in theory, empirical research, and policy." In R. D. Robinson (Editor), The International Communication of Technology: A Book of Readings: 199-223, New York: Taylor \& Francis.

Sarker, S. et al. 2005. "KT in virtual systems development teams: an exploratory study of four enablers." IEEE Transactions on Professional Communication 48(2): 201-218.

Schein, E. 1985. "Organizational Culture and Leadership.” Jossey-Bass, San Francisco, CA.

Schilling, M.A., and C.C. Phelps. 2007. "Interfirm Collaboration Networks: The Impact of Large-Scale Network Structure on Firm Innovation." Management Science 53 (7): 1113-1126.

Simon, H. 1976. "Administrative behaviour." Free Press: New York

Simonin, B. 1997. "The importance of developing collaborative know-how: An empirical test of the learning organization." Academy of Management Journal 40 (5): 1150-1174.

Song, J. et al. 2003. "Learning by hiring: when is mobility more likely to facilitate interfirm KT." Management Science 49: 351-65.

Spender, J. 1996. "Competitive advantage from tacit knowledge? Unpacking the concept and its strategic implications." In Bertrand Moingeon \& Amy Edmondson (eds.), Organisational learning and competitive advantage. London: Sage Publications.

Srai, J.S., and M. Gregory. 2008. "A Supply Network Configuration Perspective on International Supply Chain Development." International Journal of Operations and Production Management 28 (5): 386-411.

Srai, J.S., T.S. Harrington, and M.K, Tiwari. 2016. "Characteristics of redistributed manufacturing systems: a comparative study of emerging industry supply networks." International Journal of Production Research 54 (23): 6936-6955.

Szulanski, G. 1996. "Exploring internal stickiness: Impediments to the transfer of best practice within the firm." Strategic Management Journal 17: 27-43.

Szulanski, G. 2003. "Sticky knowledge: Barriers to knowing in the firm." London: Sage.

Triandis, H.C. 1995. "Individualism and Collectivism." Boulder: Westview Press.

Tushman, M. 1977. "Special boundary roles in the innovation process." Administrative Science Quarterly 22: 587-605.

Utterback, J.M. and W.J. Abernathy. 1975. "A dynamic model of process and product innovation." Omega 3 (6): 639-656. 
Utterback, J.M. 1994. "Mastering the dynamics of innovation." Boston: Harvard Business School Press.

Uzzi, B. 1996. "Sources and consequences of embeddedness for the economic performance of organizations." American Sociological Review, 61: 674-698.

Van Waarden, F. 1992. "Dimensions and types of policy networks." European Journal of Political Research, 21 (1-2): 29- 52.

Von Hippel, E. 1988. "Sources of innovation." New York: Oxford University Press.

Von Krogh, G., and J. Roos, J. 1995 "A perspective on knowledge, competence and strategy." Personal Review 24 (3): 56-76.

Wadhwa, S., and A. Saxena. 2007. "Decision knowledge sharing: flexible supply chains in KM context." Production Planning and Control 18 (5): 436-452

Wheeler, C. 2001. "Search, sorting, and urban agglomeration." Journal of Labor Economics 19(4): 879- 899.

Wiig, K. 1999. "Comprehensive KM." Knowledge Research Institute, Inc. Working Paper KRI \#1999-4 Revision 2.

Wang, F., and J. Plaskoff. 2002. "An integrated development model for KM." In R. Bellaver and J. M. Lusa (eds.), KM: Strategy and Technology. Norwood: Artech House.

Yang, J. 2008. "Antecedents and consequences of knowledge management strategy: the case of Chinese high technology firms." Production Planning and Control 19 (1): 67 77

Yeung, A. K., et al. 1999. "Organizational learning capability." New York: Oxford University Press.

Yin, R. 2003. "Case Study Research - Design and Methods." 3rd Edition. California: Sage Publications.

Zahra, S. A., and G. George. 2002. "International entrepreneurship: The current status of the field and future research agenda." In M. A. Hitt, R. D. Ireland, S. M. Camp, \& D. L. Sexton (Eds), Strategic entrepreneurship: Creating an integrated mindset: 255-288. Oxford: Blackwell.

Zander, U., and B. Kogut. 1995. "Knowledge and the speed of the transfer and imitation of organizational capabilities: An empirical test." Organization Science 6 (1): 76-92.

Zander, I., and O. Solvell. 2000. "Cross-border innovation in the multinational corporation: a research agenda." International Studies of Management and Organization 30: 44-67.

Zhang, Y., M. Gregory, and A. Neely. 2016. "Global engineering services: Shedding light on network capabilities." Journal of Operations Management (in press) http://dx.doi.org/10.1016/j.jom.2016.03.006

Zhao, H., and Y. Luo. 2005. "Antecedents of KS with peer subsidiaries in other countries: a perspective from subsidiary managers in a foreign emerging market." Management International Review 45: 71-97. 